\title{
Charge symmetry of the nuclear force as off-shell constraint*
}

\author{
P. U. Sauer ${ }^{\dagger}$ \\ Laboratory for Nuclear Science and Department of Physics, Massachusetts Institute of Technology, Cambridge, Massachusetts 02139
}

(Received 19 August 1974)

Off-shell changes are generated in the ${ }^{1} S_{0}$ nucleon-nucleon interaction using the Reid soft-core potential and unitary transformations of short range. Charge symmetry is assumed for the nuclear force. The same off-shell variations of the Reid potential are employed as the hadronic part of the proton-proton interaction and as neutron-neutron interaction. The Reid potential fits the experimental proton-proton data. It also accounts for the neutron-neutron scattering length with satisfying accuracy. The off-shell behavior of the Reid potential is varied in two different ways. First, off-shell changes are performed which preserve the fit to the proton-proton data. Most transformed potentials of the type attempted here are unable to yield the correct experimental value of the neutron-neutron scattering length and have to be rejected. A simple practical rule is given according to which the off-shell changes consistent with the neutron-neutron scattering length can be selected. Second, off-shell changes are performed which leave the neutron-neutron scattering length unaltered. Transformed potentials of this type have usually been employed in nuclear-structure calculations. The potentials which exhibit large off-shell effects in nuclear structure are unable to account for the experimental proton-proton data. Their off-shell effects are therefore of no physical significance, and the potentials have to be rejected. A simple practical rule is given according to which the off-shell changes consistent with the experimental proton-proton data can be selected.

\section{INTRODUCTION}

Microscopic nuclear-structure calculations require the knowledge of the two-nucleon interaction, on shell and off shell. Since the experimental and theoretical information on the nucleon-nucleon interaction is incomplete, significant parts of the nuclear force have to be parametrized without real physical guidance. The parametrization can be performed by continuing the two-nucleon transition matrix ${ }^{1-3}$ off the energy shell or by fitting a potential model to the experimental two-nucleon data. Both approaches allow the parametrization of the unknown parts of the nuclear force to be varied $^{4}$ keeping the fit to experiment exactly unchanged. The dependence of current nuclearstructure results on the choice of the parametrization has been demonstrated. The dependence is quite strong for nuclear matter,$^{5-8}$ the ground state of finite nuclei, ${ }^{8,9}$ and the shell-model spectra. ${ }^{8,10}$ It appears much smaller for the properties of the three-nucleon bound state. ${ }^{11,12}$

Nuclear-structure results that are sensitive with respect to changes in the parametrization of the nuclear force are interesting in themselves. They are not useful yet for determining the unknown force parameters, since the basic many-body theory underlying the results - with the exception of the three-body system -is not established beyond doubt. And even if the reliability of the theory and the accuracy of the computations were proven, it will still remain difficult to extract detailed information about the nucleon-nucleon inter- action from the results of lengthy calculations. The nuclear force, whose potential representation can become nonlocal at small distances, contains too vast a number of degrees of freedom to be easily determined this way.

As long as the nucleon-nucleon interaction remains largely unknown, constraints on its parametrization, which can simply be implemented but are physically well motivated, are badly needed. The subject of this paper is such a constraint for the isospin-triplet partial waves. It is derived from the isospin symmetries of the nuclear force. The physically most important partial wave to which the constraint applies is ${ }^{1} S_{0}$. It is also the only one for which experimental data exist in all charge states. In the following we restrict ourselves to the ${ }^{1} S_{0}$ partial wave.

The constraint is based on the simplifying as sumptions: (i) Charge symmetry holds exactly for the nucleon-nucleon interaction, i.e., the hadronic part of the proton-proton $(p p)$ and neutronneutron $(n n)$ forces are the same; and (ii) all electromagnetic effects appear in the $p p$ system and are solely due to the Coulomb force. According to this model two neutrons interact via the nuclear force only. Thus, $n n$ scattering yields direct information on the nuclear on-shell part. In contrast, $p p$ scattering is not simply determined by the on-shell part of the nucleon-nucleon interaction. $p p$ scattering arises from the interference of the nuclear force with the Coulomb interaction and therefore partially depends on the nuclear offshell behavior. The requirement that the same 
nuclear force accounts both for the experimental $n n$ scattering data and together with the Coulomb force for the experimental $p p$ scattering data places a constraint on the parametrization of the nucleon-nucleon interaction. The effectiveness of this constraint is explored in this paper.

Are the assumptions (i) and (ii) about the nuclear force well founded? Surely, charge symmetry is broken for real nucleons and, though the Coulomb force is by far the dominant electromagnetic interaction, vacuum polarization, the magnetic-moment interaction, and the neutron-proton $(n p)$ mass splitting add to the experimental differences ${ }^{13}$ between $p p$ and $n n$ scattering. However, in view of the vast uncertainty in the hadronic part of the nuclear force, the idealized force model of exact charge symmetry and of a simplified electromagnetic interaction between the protons appears to be a very useful working hypothesis.

Can the charge-symmetry constraint work effectively to narrow the arbitrariness in the parametrization of the nuclear force? One might be doubtful. Whereas $p p$ experiments have been performed with great precision at a variety of energies, the experimental $n n$ data are still very scarce. They consist essentially of the ${ }^{1} S_{0}$ effective-range parameters, scattering length $a_{n n}$ and effective range $r_{n n}$, and their best values, ${ }^{14}$

$$
a_{n n}=-16.4 \pm 0.9 \mathrm{fm}, \quad r_{n n}=2.8 \pm 0.5 \mathrm{fm},
$$

are still plagued with large uncertainties. The uncertainty of the effective range is so large that $r_{n n}$ appears really undetermined. Nevertheless, as we shall demonstrate, even the single-numbered $n n$ information on the scattering length $a_{n n}$ can rule out a variety of off-shell parametrizations of the ${ }^{1} S_{0}$ nucleon-nucleon interaction. $\mathrm{Ob}-$ viously, a comparison of the $p p$ data with the equally rich $n p$ data in ${ }^{1} S_{0}$ could be done in much more detail. But such a comparison would have to be based on the charge independence of the nuclear force and charge independence is not as justified $^{15}$ as charge symmetry. The difference between the $p p$ and $n p$ interactions does not solely arise from known direct electromagnetic effects, but also from indirect ones, e.g., the charge dependence of the exchanged mesons. The chargedependent part of the nuclear force, however, is as little known as the complete nuclear force itself. Only if the violation of charge independence is specified on shell and off shell can the comparison between $p p$ and $n p$ scattering yield additional information on the off-shell behavior of the chargeindependent part of the nucleon-nucleon interaction. This is the reason why we remain content in this study to exploit the effect of charge symmetry as an off-shell constraint in the ${ }^{1} S_{0}$ partial wave.
The effect of charge symmetry as a constraint for the parametrization of the nucleon-nucleon interaction is studied in the context of potential models. The same investigation using the two-nucleon transition matrix directly is underway. ${ }^{16}$ Here, we start out from the Reid soft-core potential ${ }^{17}$ $V_{R}$ which accounts together with the Coulomb force for the experimental $p p$ scattering data and also for the $n n$ data (1) with satisfying accuracy. Using the technique of short-ranged unitary transformations ${ }^{4}$ the potential $V_{R}$ is varied in two ways.

First, in Sec. II families of nuclear $p p$ potentials are generated which together with the Coulomb force reproduce the experimental $p p$ data as exactly as $V_{R}$ at all energies. Assuming charge symmetry, the transformed potentials are also used as $n n$ potentials. A great number of them turn out to be inconsistent with the $n n$ data (1). They are therefore judged as not acceptable. A simple rule is given that allows one to sort out these unacceptable potential parametrizations without lengthy calculations.

Second, in Sec. III families of potentials are generated which are exactly equivalent with respect to purely nuclear on-shell scattering. By construction they all account for the $n n$ data as well as the Reid potential does. However, many do not fit the $p p$ data and therefore have to be ruled out. Transformed potentials of this type have usually been employed in nuclear-structure calculations. ${ }^{4-7,9-11}$ A simple practical rule is given that allows one to sort out the unacceptable potentials.

Both methods are complementary and simply approach the charge-symmetry constraint on the off-shell parametrization of the isospin-triplet nucleon-nucleon potential from opposite directions.

\section{II. ${ }^{1} S_{0}$ NUCLEON-NUCLEON POTENTIALS EQUIVALENT WITH RESPECT TO THE EXPERIMENTAL PROTON-PROTON DATA}

\section{A. How are appropriate off-shell changes generated?}

In this section ${ }^{1} S_{0}$ nuclear potential models are generated that are equivalent to each other and to the local soft-core Reid potential $V_{R}$. All potentials must have the theoretically required local one-pion exchange tail and must account, together with the Coulomb force $V_{C}$, for the experimental ${ }^{1} S_{0} p p$ phase shift in exactly the same way.

The nuclear potentials $\tilde{V}_{R}$,

$$
\tilde{V}_{R}=U\left(K+V_{C}+V_{R}\right) U^{\dagger}-K-V_{C},
$$

have the desired properties by construction. In Eq. (2), $K$ denotes the kinetic energy operator of relative motion (the corresponding momentum is 
$k$ ), and $U$ is an arbitrary unitary operator of short range.

(i) Together with the Coulomb potential they yield the same phase $\eta_{p p R}^{c}(k)$ as the Reid potential at all energies. The scattering states $|\bar{\phi}(k)\rangle$ of $\tilde{V}_{R}$ are related to those of $V_{R},|\phi(k)\rangle$, by $|\tilde{\phi}(k)\rangle$ $=U|\phi(k)\rangle$, and both $|\tilde{\phi}(k)\rangle$ and $|\phi(k)\rangle$ take on the

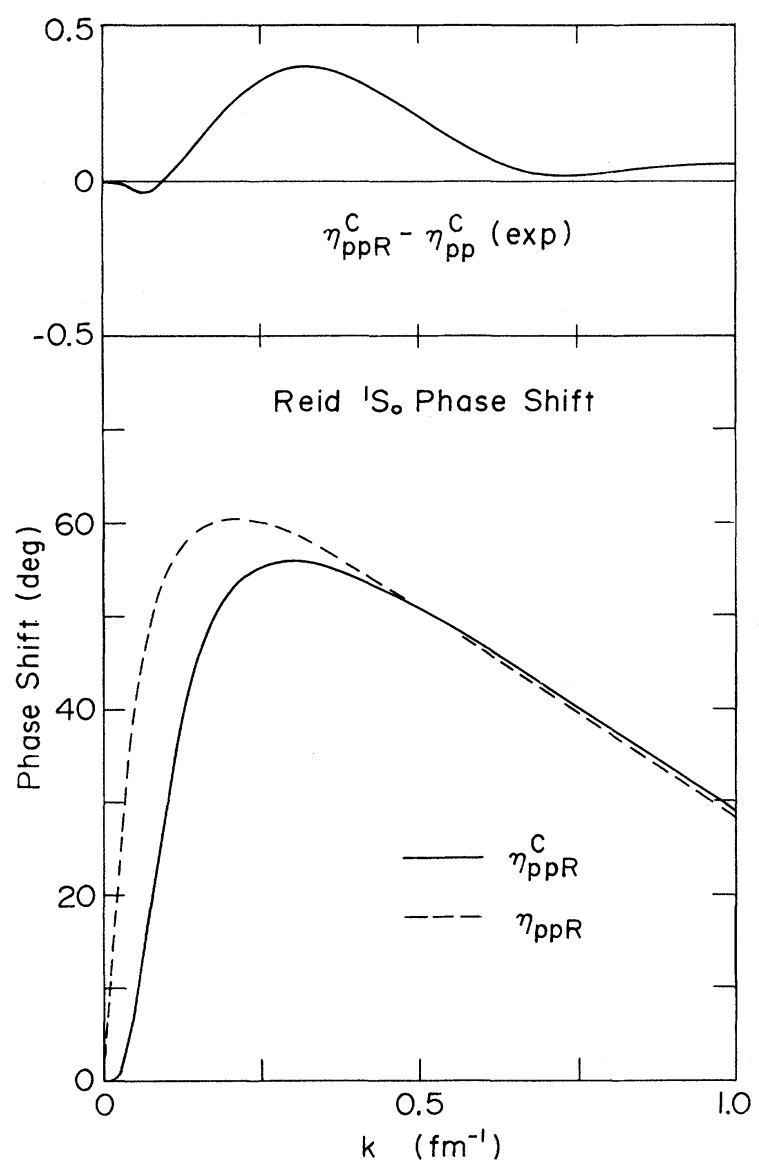

FIG. 1. Phase shift of the ${ }^{1} S_{0}$ Reid soft-core potential. The phase shift with Coulomb is denoted by $\eta_{p p R}^{C}$. It is the same for the Reid potential and the potentials $\tilde{V}_{R}$ defined in Eq. (2). The effective-range parameters corresponding to $\eta_{p p R}^{C}$ are $a_{p p R}^{C}=-7.78 \mathrm{fm}$ and $r_{p p R}^{C}=2.72$ $\mathrm{fm}$. The point Coulomb potential is used for the results of this figure. The high quality of the fit of the Reid potential to the experimental $p p$ phase shift $\eta_{p p}^{C}$ (exp) is shown in the upper part of the figure, where the phase shift difference $\eta_{p p R}^{C}-\eta_{p p}^{C}$ (exp) is plotted. The experimental phase shift is taken from Ref. 19, its effectiverange parameters (Ref. 20) are $a_{p p}^{c}=-7.823 \pm 0.01 \mathrm{fm}$ and $r_{p p}^{C}=2.794 \pm 015 \mathrm{fm}$. The Coulomb-subtracted purely nuclear phase shift of the Reid potential is also shown and denoted by $\eta_{p p R}$ in the figure. The purely nuclear effective-range parameters are $a_{p p R}=-17.2 \mathrm{fm}$ and $r_{p p R}=2.80 \mathrm{fm}$. All phase shifts are shown as function of the relative momentum $k$. The maximum momentum 1 $\mathrm{fm}^{-1}$ corresponds to $83 \mathrm{MeV}$ energy in the lab system. same asymptotic form $\left\langle r \mid \phi_{a}(k)\right\rangle$,

$$
\begin{aligned}
& \left\langle r \mid \phi_{a}(k)\right\rangle=C_{0}(\gamma)\left[\cot \eta_{p p R}^{c}(k) F_{0}(\gamma, k r)+G_{0}(\gamma, k r)\right], \\
& C_{0}{ }^{2}(\gamma)=2 \pi \gamma /\left[e^{2 \pi \gamma}-1\right], \quad \gamma=e^{2} M /\left(2 \hbar^{2} k\right) .
\end{aligned}
$$

In Eq. (3) $F_{0}\left(G_{0}\right)$ is the regular (irregular) Coulomb wave function ${ }^{18}$ of orbital momentum $0, r$ is the $p p$ distance, and $M(e)$ is the proton mass (charge). The phase shift $\eta_{p p R}^{C}(k)$ is displayed in Fig. 1. It is calculated with the point Coulomb interaction $V_{C}^{P}=e^{2} / r$. It matches the experimental one ${ }^{19}$ with satisfying accuracy. ${ }^{17}$ Its scattering length $a_{p p R}^{C}$, obtained from the effective-range expansion ${ }^{13,17}$ of the phase shift $\eta_{p p R}^{C}(k)$,

$C_{0}{ }^{2}(\gamma) k \cot \eta_{p p R}^{C}(k)+2 k \gamma h(\gamma)=-\frac{1}{a_{p p R}^{C}}+\frac{1}{2} r_{p p R}^{c} k^{2}+\mathcal{O}\left(k^{4}\right)$,

is $-7.78 \mathrm{fm}$ and is only slightly less attractive than the experimental value ${ }^{20}$ of $-7.823 \pm 0.01 \mathrm{fm}$. Its effective range $r_{p p R}^{c}$ is $2.72 \mathrm{fm}$ and differs quite seriously from the experimental value ${ }^{20}$ of 2.794 $\pm 0.015 \mathrm{fm}$. Nevertheless, the Reid potential $V_{R}$ and the potentials $\tilde{V}_{R}$ represent identical and, for our purposes, sufficiently good fits to the experimental ${ }^{1} S_{0} p p$ data. The function $h(\gamma)$ needed for Eq. (4) is defined as follows:

$$
h(\gamma)=\sum_{n=1}^{\infty} \frac{\gamma^{2}}{n\left(n^{2}+\gamma^{2}\right)}-\ln \gamma-\gamma_{E},
$$

with $\gamma_{E}$ being Euler's constant 0.5772.

(ii) The long-range Coulomb potential $V_{C}$ appears in Eq. (2) only to guarantee the same $p p$ phase $\eta_{p p R}^{C}(k)$ for all $\tilde{V}_{R}$. The potentials $\tilde{V}_{R}$ are purely nuclear without Coulomb. They contain neither off-shell changes of the known Coulomb force nor other unwanted electromagnetic effects. The potentials $\tilde{V}_{R}$ are nonlocal at small distances, but reduce to the local tail of $V_{R}$ where $U$ reduces to unity. The simple for $\mathrm{m}^{6}$

$$
\begin{aligned}
& U=1-2|g\rangle\langle g|, \\
& \langle r \mid g\rangle=C r(1-\beta r) e^{-\alpha r}
\end{aligned}
$$

is employed in this paper. With the extra factor $r$ included in Eq. (6) the radial part of the volume element for integration is $d r$. The constant $C$ ensures the normalization of $|g\rangle$. The range of $U$ is controlled by $\alpha$. The potentials $\tilde{V}_{R}$ have the onepion exchange tail of $V_{R}$ as required by theory, if $\alpha$ is chosen sufficiently large.

Except for Ref. 21, the present method (2) of generating off-shell changes in the nucleon-nucleon interaction has not been tried before. Instead of $\tilde{V}_{R}$, the potential models $U\left(K+V_{R}\right) U^{\dagger}-K$ have usually been used in nuclear-structure calculations ${ }^{4-7,9-11}$ to study off -shell effects. They 
preserve the purely nuclear, i.e., Coulomb-subtracted phase $\eta_{p p R}(k)$, which is not an observable in $p p$ scattering and which differs substantially from the experimental phase and $\eta_{p p R}^{c}(k)$ at low energies. The latter fact is demonstrated in Fig. 1 for the Reid potential. We shall return to the potential models $U\left(K+V_{R}\right) U^{\dagger}-K$ in Sec. III. In contrast to them the method of Eq. (2) for generating off-shell changes in the nuclear potential keeps the fit of $V_{R}$ to the experimental $p p$ data unaltered and is therefore clearly preferable.

B. Do the potentials $\tilde{V}_{R}$ account for the $n n$ scattering length?

In this section the Coulomb-subtracted Hamiltonian $K+\tilde{V}_{R}$ is studied. Its Schrödinger equation is solved in momentum space. ${ }^{22}$ The resulting scattering states $|\tilde{\psi}(k)\rangle$ have the asymptotic form $\left\langle r \mid \psi_{a}(k)\right\rangle$,

$$
\left\langle r \mid \psi_{a}(k)\right\rangle=\cot \eta_{p p}(k) \sin k r+\cos k r,
$$

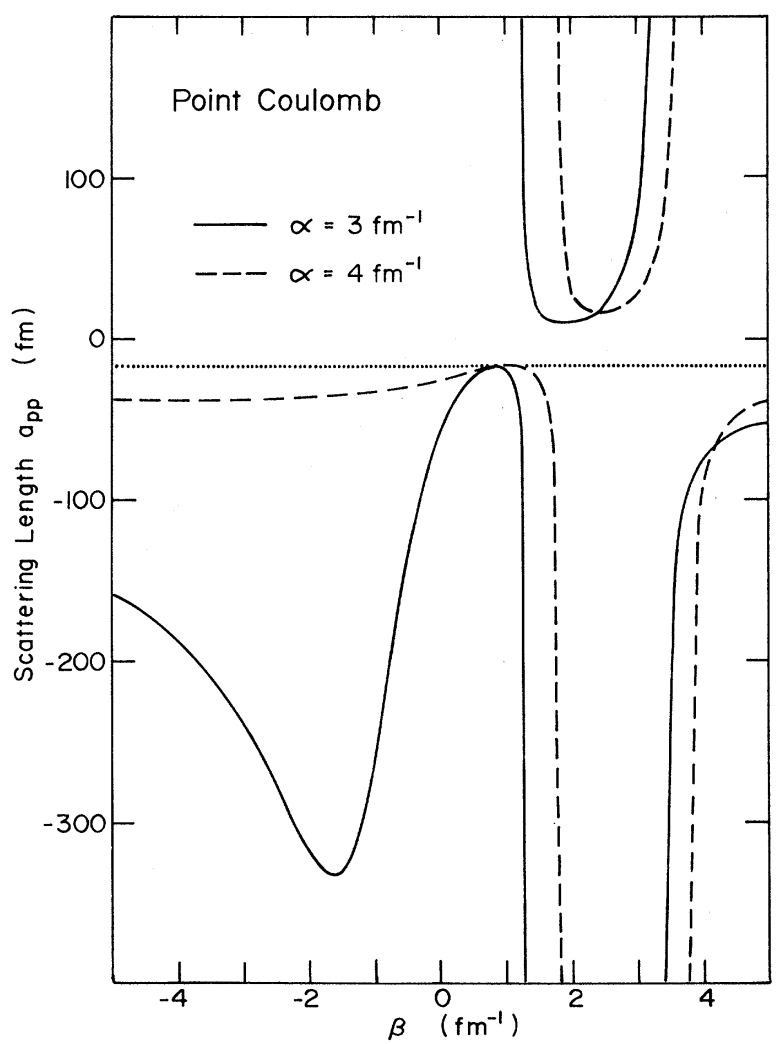

FIG. 2. Scattering length $a_{p p}$ of the Coulomb-subtracted $p p$ phase shifts $\eta_{p p}(k)$ resulting from the potentials $\tilde{V}_{R}$. The point Coulomb potential is taken for $V_{C}$. The unitary transformation (6) is used with two different range parameters $\alpha$. The results are shown as function of the parameter $\beta$. The corresponding scattering length $a_{p p R}$ of the untransformed Reid potential is $-17.2 \mathrm{fm}$. The experimental value (1) of the $n n$ scattering length is indicated by the dotted line.

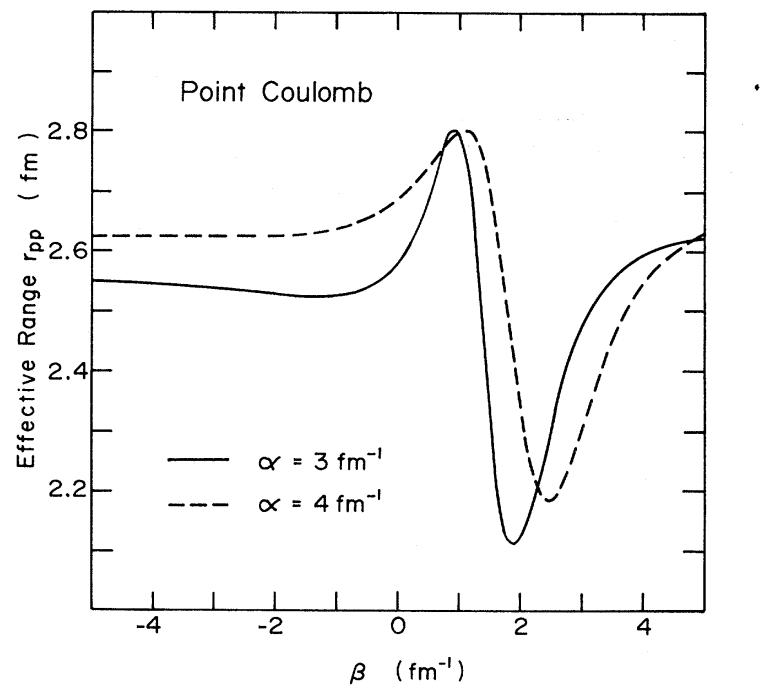

FIG. 3. Effective range $r_{p p}$ of the Coulomb-subtracted $p p$ phase shifts $\eta_{p p}(k)$ resulting from the potentials $\tilde{V}_{R}$. The point Coulomb potential is taken for $V_{C}$. The unitary transformation (6) is used with two different range parameters $\alpha$. The results are shown as function of the parameter $\beta$. The corresponding effective range $r_{p p R}$ of the untransformed Reid potential is $2.80 \mathrm{fm}$. The experimental value (1) of the $n n$ effective range is not indicated, its error is still too large.

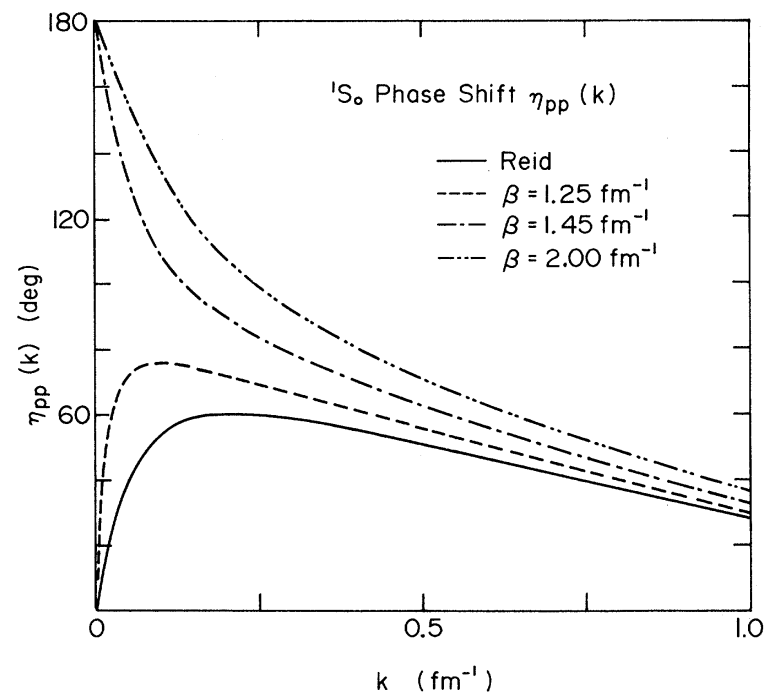

FIG. 4. Coulomb-subtracted $p p$ phase shifts $\eta_{p p}(k)$ resulting from selected potentials $\tilde{V}_{R}$. The point Coulomb potential is taken for $V_{C}$. The unitary transformation (6) is used with $\alpha$ being $3 \mathrm{fm}^{-1}$ and with three different $\beta$ values, $1.25,1.45$, and $2.00 \mathrm{fm}^{-1}$. The corresponding phase shift $\eta_{p p}(k)$ of the untransformed Reid potential is also shown. The phase shifts are plotted as function of the relative momentum $k$. The maximum momentum $1 \mathrm{fm}^{-1}$ corresponds to $83 \mathrm{MeV}$ energy in the lab system. 
which yields the purely nuclear $p p$ phase shift $\eta_{p p}(k)$. The effective-range parameters of $\eta_{p p}(k)$, scattering length $a_{p p}$ and effective range $r_{p p}$, are obtained from the low-energy expansion of $\eta_{p p}(k)$ :

$$
k \cot \eta_{p p}(k)=-\frac{1}{a_{p p}}+\frac{1}{2} r_{p p} k^{2}+\mathcal{O}\left(k^{4}\right) .
$$

The phase shift $\eta_{p p}(k)$ is not an observable. The assumption of charge symmetry, however, equates $\eta_{p p}(k)$ with the observable $n n$ phase shift $\eta_{n n}(k)$. Unfortunately, only the scattering length $a_{n n}$ of $\eta_{n n}(k)$ has been reliably measured. How well do the potentials $\tilde{V}_{R}$ account for the experimental value (1) of $a_{n n}$ ? This is the question we want to answer when calculating $\eta_{p p}(k)$ in the present section.

Since the Reid potential is fitted to yield the experimental $p p$ data together with the point Coulomb potential, the point Coulomb potential $V_{C}^{P}$ is also used for $V_{C}$ in Eq. (2). The Coulomb-subtracted $p p$ scattering length $a_{p p}$ resulting from $\tilde{V}_{R}$ is displayed in Fig. 2 for a wide range of parameters in the unitary transformation $U$. The corresponding effective range $r_{p p}$ is given in Fig. 3. Figure 4 shows the phase shifts $\eta_{p p}(k)$ themselves for selected parameter sets. Though the phase shift $\eta_{p p R}^{c}(k)$ is exactly the same for the potentials $\tilde{V}_{R}$, the nuclear phase shifts $\eta_{p p}(k)$ and their effectiverange parameters do not at all stay the same but depend strongly on $\tilde{V}_{R}$. The scattering length $a_{p p}$ exhibits the most dramatic variation with $\tilde{V}_{R}$. As can be read off from Fig. 2, only potentials obtained from transformations $U$ within a very small parameter range around $\beta=0.9 \mathrm{fm}^{-1}$ for $\alpha=3 \mathrm{fm}^{-1}$ and around $\beta=1.1 \mathrm{fm}^{-1}$ for $\alpha=4 \mathrm{fm}^{-1}$ are consistent with $a_{n n}$. Most other potentials $\tilde{V}_{R}$ are unable to account for $a_{n n}$ with satisfying accuracy. They therefore have to be rejected. The spread in the results is smaller for the effective range and the low-energy phase shift, but it remains sizable even for them. For the latter quantities, however, no corresponding $n n$ data are presently available to yield additional charge-symmetry constraints.

The theoretical subtraction of the Coulomb ef fects from the experimental $p p$ data is a strongly potential-dependent procedure. This is surprising in view of the fact, that the Coulomb interaction is known and much weaker than the nuclear force. What is the reason for the strong potential dependence? One might suspect, that for the $\tilde{V}_{R}$ the Coulomb subtraction exploits the unphysical $1 / r$ singularity of $V_{C}^{P}$ in an improper way. If this were the case, the Coulomb potential should be appropriately modified for the finite size of the proton, and the finite-size ${ }^{23}$ Coulomb potential $V_{C}^{F}$, i.e.,

$$
V_{C}^{F}(r)=\frac{e^{2}}{r}\left[1-e^{-x}\left(1+\frac{11}{16} x+\frac{3}{16} x^{2}+\frac{1}{48} x^{3}\right)\right]
$$

should be used in Eq. (2) instead of $V_{C}^{P}$. In Eq. (9) $x=\sqrt{12} r / R_{p}$ and $R_{p}$ denotes the proton $\mathrm{rms}$ radius. In Fig. 5, the Coulomb-subtracted effective-range parameters $a_{p p}$ and $r_{p p}$ are therefore recomputed as function of the proton size $R_{p}$ for one selected potential $\tilde{V}_{R}$ arising from the unitary transformation (6) with $\alpha=3 \mathrm{fm}^{-1}$ and $\beta=2 \mathrm{fm}^{-1}$. Indeed, the results depend strongly on $R_{p}$. Their deviation from the corresponding Reid values, $a_{p p R}=-17.2$ $\mathrm{fm}$ and $r_{p p R}=2.80 \mathrm{fm}$, is largest for the point Coulomb potential and decreases rapidly with increasing $R_{p}$. This trend confirms the dominating role of the $1 / r$ singularity for the results of Figs. 2-4. It forces us to repeat the Coulomb subtraction using the more physical Coulomb potential $V_{C}^{\boldsymbol{F}}$ with the experimental proton size $R_{p}=0.8 \mathrm{fm}$ in Eq. (2). The Coulomb potentials $V_{C}^{P}$ and $V_{C}^{F}$ are compared in Fig. 6. Figure 6 also demonstrates that together with $V_{C}^{F}$ the Reid potential $V_{R}$ and the potentials $\tilde{V}_{R}$ of Eq. (2) still reproduce the experimental $p p$ data $^{17,19}$ well; though the quality of the fit has slightly deteriorated. We observe in the displayed errors of Fig. 6 that $V_{R}$ and $\tilde{V}_{R}$ with $V_{C}^{P}$ are too repulsive at low energies, whereas with $V_{C}^{F}$ they are too attractive. E.g., the scattering length of $\eta_{p p R}^{C}(k)$ with $V_{C}^{F}$ is $-7.89 \mathrm{fm}$, it is by about $1 \%$ too

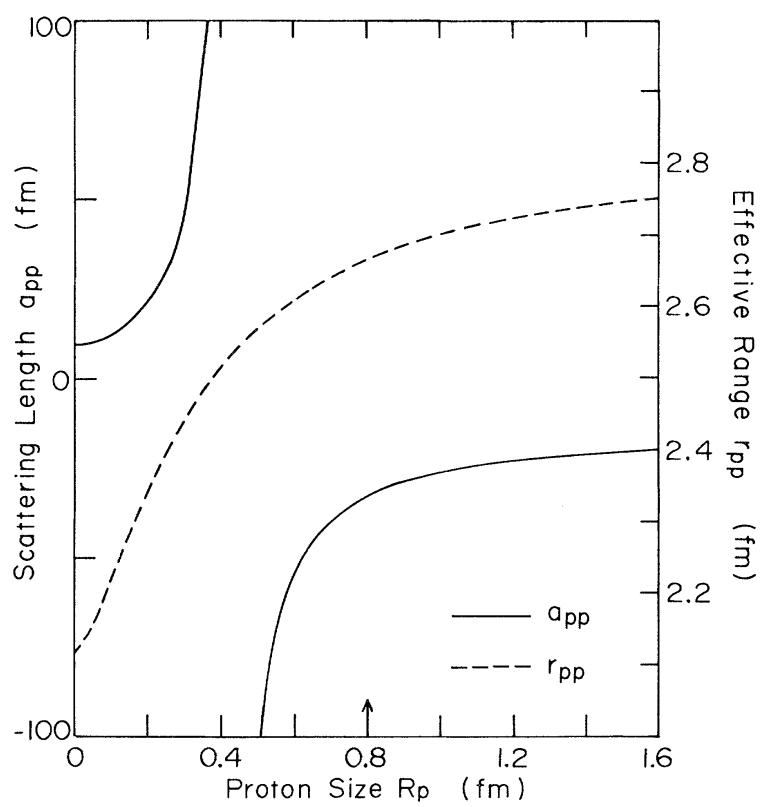

FIG. 5. Effective-range parameters $a_{p p}$ and $\gamma_{p p}$ of the Coulomb-subtracted $p p$ phase shift $\eta_{p p}(k)$. Results are given for the potential $\tilde{V}_{R}$ using $\alpha=3 \mathrm{fm}^{-1}$ and $\beta=2.0$ $\mathrm{fm}^{-1}$ in the unitary transformation (6). The finite-size Coulomb potential is taken for $V_{C}$. The effective-range parameters are shown as function of the proton size $R_{p}$ The experimental proton size $0.8 \mathrm{fm}$ is indicated by the arrow. 
large in magnitude.

The Coulomb subtraction in the ${ }^{1} S_{0} p p$ data is repeated for the potentials $\tilde{V}_{R}$ using $V_{C}^{\boldsymbol{F}}$. The results for the effective-range parameters are given in Figs. 7 and 8 . As compared to the corresponding results of Figs. 2 and 3 with $V_{C}^{P}$, the spread in the results is generally much reduced consistent with the trend found in Fig. 5 for a particular potential $\tilde{V}_{R}$. However, the variation in the results
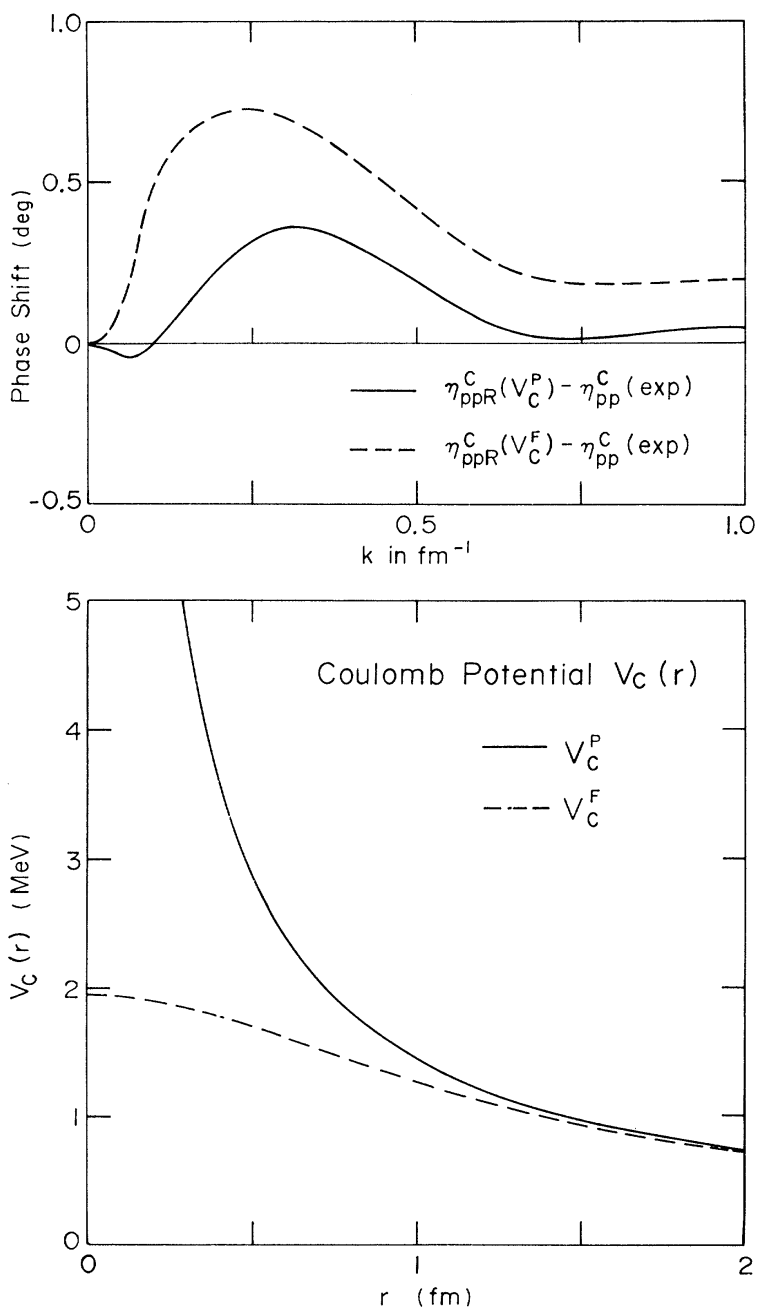

FIG. 6. Comparison of the point Coulomb $\left(V_{C}^{P}\right)$ and the finite-size Coulomb $\left(V_{C}^{F}\right)$ potentials. The experimental proton size $0.8 \mathrm{fm}$ is used in $V_{C}^{F}$. The quality of the fit of the ${ }^{1} S_{0}$ Reid soft-core potential to the experimental $p p$ phase shift $\eta_{p p}^{C}$ (exp) is shown in the upper part of the figure. The Reid phase shifts are obtained with the point Coulomb and the finite-size Coulomb potentials and are denoted by $\eta_{p p R}^{C}\left(V_{C}^{P}\right)$ and $\eta_{p p R}^{C}\left(V_{C}^{F}\right)$, respectively. Their deviation from the experimental phase shift is plotted as function of the relative momentum $k$. The maximum momentum $1 \mathrm{fm}^{-1}$ corresponds to $83 \mathrm{MeV}$ energy in the lab system. still remains substantial. The subtraction of Coulomb effects from the experimental $p p$ data strongly depends on the parametrization of the theoretically unknown short-distance part of the nuclear force. From this we had now to conclude, as in Ref. 24, that the charge symmetry of the nuclear force cannot be confirmed by nucleon-nucleon scattering experiments in a model-independent way. However, in contrast to Ref. 24 we have assumed charge symmetry to hold and use the potentials $\tilde{V}_{R}$ as $n n$ potentials. The $n n$ potentials $\tilde{V}_{R}$, except those within a small parameter range around $\beta=0.9 \mathrm{fm}^{-1}$ for $\alpha=3 \mathrm{fm}^{-1}$ and around $\beta$ $=1.1 \mathrm{fm}^{-1}$ for $\alpha=4 \mathrm{fm}^{-1}$, are unable to account for the experimental $n n$ scattering length. Thus, most $\tilde{V}_{R}$ are unacceptable $n n$ potentials and have to be discarded; though they account well for all $p p$ data. Only if a sizable amount of charge asymmetry were allowed in the nuclear force could a wider class of potentials $\tilde{V}_{R}$ be judged acceptable. Provided the errors in the experimental $n n$ scattering length are as reliable as quoted in Eq. (1), the requirement of charge symmetry is an effective constraint on the off-shell parametrization of the ${ }^{1} S_{0} p p$ interaction, independent of the choice of $V_{C}^{P}$ or $V_{C}^{F}$.

The charge-symmetry constraint has a beneficial theoretical side effect. Though it is based on low-energy scattering data, it constrains the parametrization of the nucleon-nucleon interaction

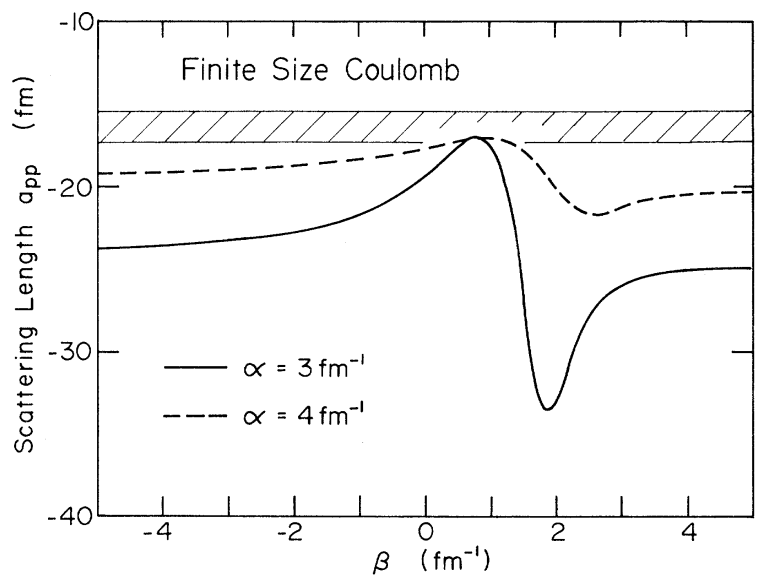

FIG. 7. Scattering length $a_{p p}$ of the Coulomb-subtracted $p p$ phase shifts $\eta_{p p}(k)$ resulting from the potentials $\tilde{V}_{R}$. The finite-size Coulomb potential is taken for $V_{C}$. The unitary transformation (6) is used with two different range parameters $\alpha$. The results are shown as function of the parameter $\beta$. The corresponding scattering length $a_{p p R}$ of the untransformed Reid potential is $-17.2 \mathrm{fm}$. The cross-hatched strip indicates the range of values consistent with the $n n$ scattering length of Eq. (1) and charge symmetry. 
in the core region. This will become evident later in Sec. IIC. Furthermore, many potentials $\tilde{V}_{R}$, which are ruled out by this constraint, would produce disastrous nuclear-structure results. Figure 9 displays some ${ }^{1} S_{0}$ reaction matrix elements appropriate for shell-model calculations in ${ }^{18} \mathrm{O}$.

Their variation with $\tilde{V}_{R}$ is very large indeed. The matrix elements are generally far more repulsive than the corresponding elements of the Reid potential $V_{R}$. This is surprising, since the purely nuclear scattering amplitude of the potentials $\tilde{V}_{R}$ shows the opposite trend according to Figs. 2-4, 7 , and 8. What is the reason? Changing the nucleon-nucleon potential from $V_{R}$ to $\tilde{V}_{R}$ affects the purely nuclear ${ }^{1} S_{0}$ phase shift $\eta_{p p}(k)$ and the wound integral. The wave functions displayed in Figs. 11 and 12 of Sec. II C indicate the latter effect on the radial shape of the short-range correlations. They correspond to increased wound integrals. According to Ref. 10 there is a simple linear relation between a decreased attraction in the shellmodel interaction of phase-equivalent potentials and an increased wound integral. However, this relation is not immediately applicable to the potentials $\tilde{V}_{R}$ and $V_{R}$, since they are not equivalent with respect to the nuclear phase $\eta_{p p}(k)$. Compared to $V_{R}, \tilde{V}_{R}$ therefore gains some additional attraction for its shell-model reaction matrix due to variations in $\eta_{p p}(k)$. On the other hand, the increase in its wound integral is generally so dramatic that the combined result is a substantial re-

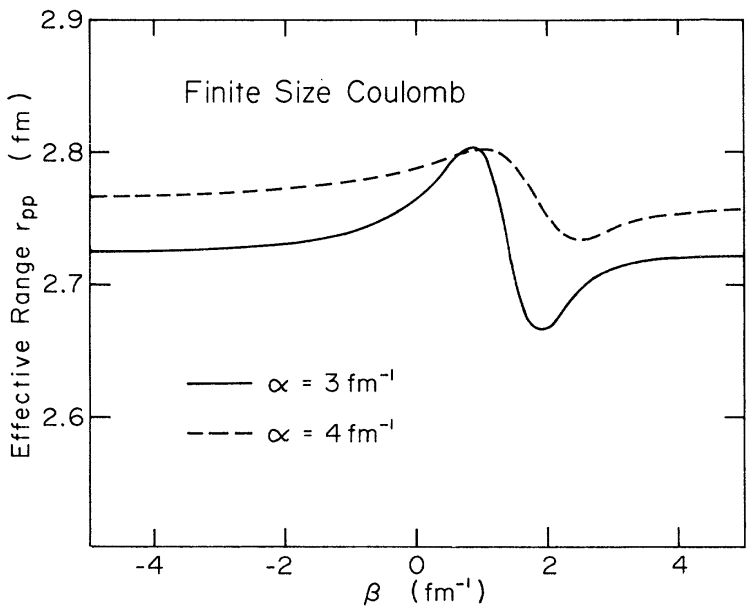

FIG. 8. Effective range $r_{p p}$ of the Coulomb-subtracted $p p$ phase shifts $\eta_{p p}(k)$ resulting from the potentials $\tilde{V}_{R}$. The finite-size Coulomb potential is taken for $V_{C}$. The unitary transformation (6) is used with two different range parameters $\alpha$. The results are shown as function of the parameter $\beta$. The corresponding effective range $r_{p p R}$ of the untransformed Reid potential is $2.80 \mathrm{fm}$. The experimental value (1) of the $n n$ effective range is not indicated; its error is still too large. pulsive shift in the shell-model interaction as shown in Fig. 9. The matrix elements of Fig. 9 are obtained with $V_{C}^{P}$ in Eq. (2), but matrix elements calculated with $V_{C}^{F}$ and with the potentials $U\left(K+V_{R}\right) U^{\dagger}-K$ of Sec. III agree with those of Fig. 9 within $1 \mathrm{MeV}$ for corresponding transformation parameters. Consistent with the trend of the purely nuclear scattering amplitude, the matrix elements of $\tilde{V}_{R}$ are usually more attractive than the

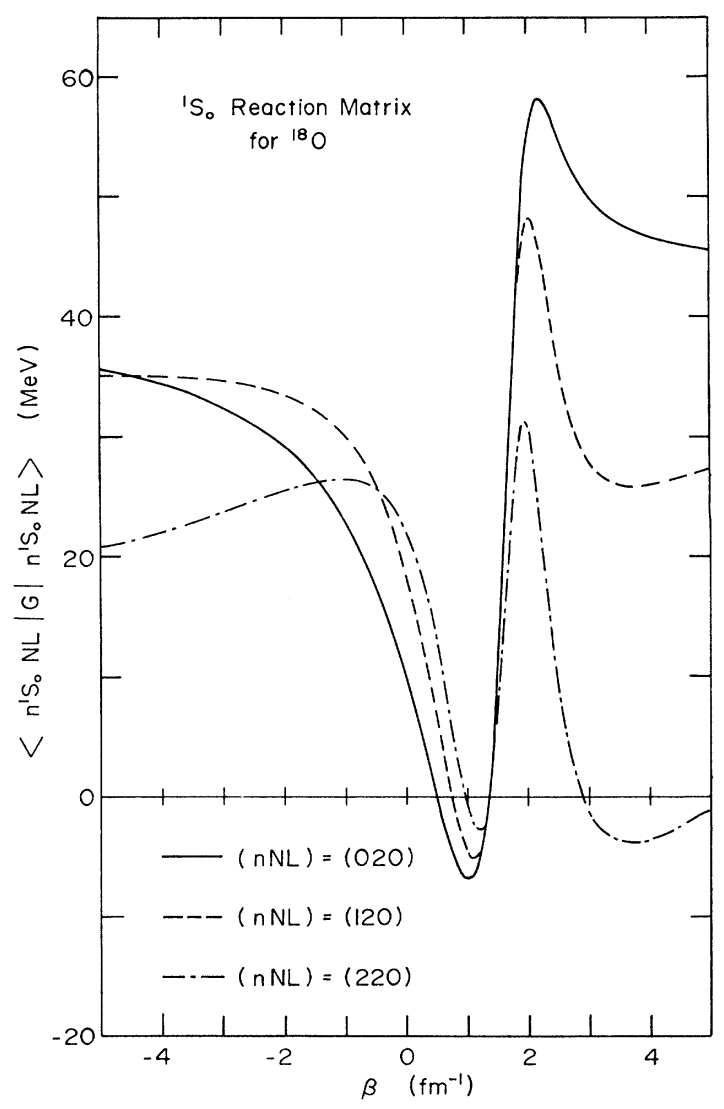

FIG. 9. Diagonal ${ }^{1} S_{0}$ reaction matrix elements $\left\langle n^{1} S_{0} N L|G| n^{1} S_{0} N L\right\rangle$ in oscillator representation for a shell-model calculation of ${ }^{18} \mathrm{O}$. The matrix elements are obtained from the potentials $\tilde{V}_{R}$ using the method of Ref. 25. The point Coulomb potential is taken for $V_{C}$. The unitary transformation (6) is used with the range parameter $\alpha$ being $3 \mathrm{fm}^{-1}$. The results are shown as function of the parameter $\beta$. In the matrix elements the available energy is taken to be $-10 \mathrm{MeV}$, the oscillator parameter is $1.72 \mathrm{fm}$. The oscillator quantum number $n$ refers to the relative motion; the radial quantum number $N$ and the orbital momentum $L$ refer to the c.m. motion. The quantum-number combination (120) and $(220)$ do not arise in computing the bare effective shell-model interaction. The matrix elements obtained with the finite-size Coulomb potential and the matrix elements obtained from the potentials $\bar{V}_{R}$ of Sec. III agree with those of the figure within the accuracy of the plot. 
corresponding ones of $U\left(K+V_{R}\right) U^{\dagger}-K$.

Since the reaction matrix elements calculated with the potentials $\tilde{V}_{R}$ and $U\left(K+V_{R}\right) U^{\dagger}-K$ are very similar, already existing ${ }^{4,6,9-11}$ nuclear-structure results with $U\left(K+V_{R}\right) U^{\dagger}-K$ should be characteristic, except in details, for results with $\tilde{V}_{R}$. Known and additionally calculated results for some potentials $U\left(K+V_{R}\right) U^{\dagger}-K$ are compiled in the table in Sec. III. Since, however, existing nuclear-structure results do not span a wide range in the transformation parameters of Eq. (6), one ${ }^{18} \mathrm{O}$ spectrum is shown in Fig. 10 as an example derived from a potential $\tilde{V}_{R}$ outside the previously explored parameter range. The spectrum looks indeed disturbingly unfamiliar. We expect similarly shocking results for other nuclear-structure calcula-

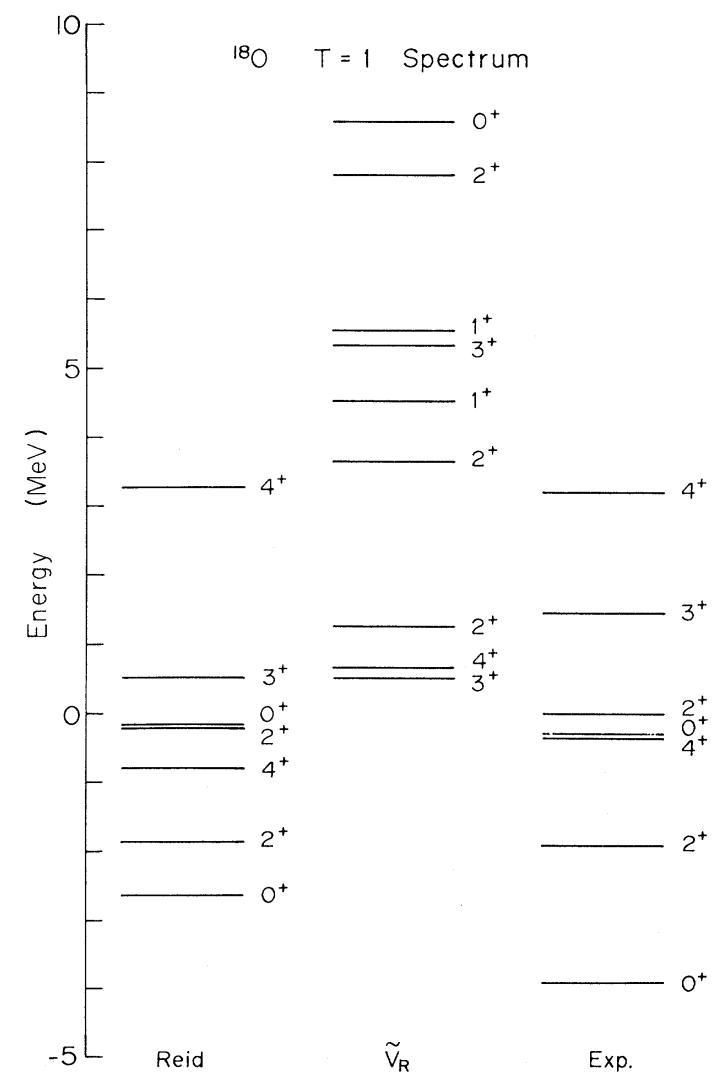

FIG. 10. Shell-model spectrum of ${ }^{18} \mathrm{O}$. The results represent a calculation with the bare effective interaction without core-polarization correction. The technical details are those of Ref. 10. The spectrum corresponding to the Reid soft-core potential is compared with a spectrum obtained by using $\tilde{V}_{R}$ as ${ }^{1} S_{0}$ potential with $\alpha=3 \mathrm{fm}^{-1}$ and $\beta=2.0 \mathrm{fm}^{-1}$ and with $V_{C}^{P}$ in Eq. (2). It is considered entirely unlikely that higher-order correction in the effective interaction shift the two spectra into better mutual agreement. The experimental spectrum is also shown. tions with the same $\tilde{V}_{R}$; though they have not been performed. But strange nuclear-structure results are an indication not an undisputable proof that the parametrization of the nucleon-nucleon interaction used is unacceptable. In contrast, the requirement of charge symmetry, which rules out the potential $\tilde{V}_{R}$ employed in Fig. 10, appears to be a clear, effective, and much simpler off-shell constraint in the ${ }^{1} S_{0}$ partial wave.

C. Can the charge-symmetry constraint easily be implemented?

In the previous section we were able to determine the acceptability of a potential $\tilde{V}_{R}$ after its Coulomb-subtracted scattering length was calculated and compared with the experimental $n n$ value (1). This is a straightforward method, much easier to use, for instance, than a decision on the nuclear force at the end of a lengthy three-body calculation. Still, we would like to select the allowed potentials $\tilde{V}_{R}$ by even simpler means. Such a simplified method of selecting the acceptable $\tilde{V}_{R}$ is the subject of this section.

First, we give an exact relation between the purely nuclear phases $\eta_{p p}(k)$ and $\eta_{p p R}(k)$ of $\tilde{V}_{R}$ and $V_{R}$, respectively. We apply the two-potential formula for the on-shell two-nucleon transition matrix to the nuclear potentials $\tilde{V}_{R}$ of Eq. (2) and $U\left(K+V_{R}\right) U^{\dagger}-K$. The latter has the same Coulombsubtracted phase shift $\eta_{p p_{R}}(k)$ as $V_{R}$. Their potential difference $U V_{C} U^{\dagger}-V_{C}$ depends on $V_{C}$, but in accordance with Sec. II A $U V_{C} U^{\dagger}-V_{C}$ has to be interpreted as the difference between two purely nuclear potentials. The two-potential formula yields

$$
\begin{aligned}
k \cot \eta_{p p}(k)= & k \cot \eta_{p p R}(k)+\frac{M}{\hbar^{2}} \\
& \times\left\langle U \psi(k)\left|U V_{C} U^{\dagger}-V_{C}\right| \tilde{\psi}(k)\right\rangle .
\end{aligned}
$$

Equation (10) is derived in Appendix A. It employs wave functions in the effective-range normalization (7). The scattering wave functions of the Reid potential $V_{R}$ are denoted by $|\psi(k)\rangle$; the wave functions of $U\left(K+V_{R}\right) U^{\dagger}-K$ are $U|\psi(k)\rangle$. Second, if $|\tilde{\psi}(k)\rangle$ could be approximated by $U|\psi(k)\rangle$, then the phase shifts $\eta_{p p}(k)$ were given in a transparent and easily discussible way,

$$
\begin{aligned}
k \cot \eta_{p p}(k) \simeq & k \cot \eta_{p p R}(k)-\frac{M}{\hbar^{2}} \\
& \times\left\langle\psi(k)\left|U^{\dagger} V_{C} U-V_{C}\right| \psi(k)\right\rangle, \\
\eta_{p p}(k)-\eta_{p p R}(k) \simeq & \frac{\sin ^{2} \eta_{p p R}(k)}{k} \frac{M}{\hbar^{2}} \\
& \times\left\langle\psi(k)\left|U^{\dagger} V_{C} U-V_{C}\right| \psi(k)\right\rangle,
\end{aligned}
$$


entirely in terms of quantities referring to the untransformed potential $V_{R}$. The approximation $|\tilde{\psi}(k)\rangle \simeq U|\psi(k)\rangle$ is a crucial one and clearly needs justification. Since the phase shifts of $|\tilde{\psi}(k)\rangle$ and $U|\psi(k)\rangle$ are different, the two wave functions differ at large distances. Nevertheless, one might expect their qualitative agreement over the range of the unitary transformation, where the character of the wave function is dominated by the transformation and where it appears not so important what terms in the Hamiltonian are transformed. Only the short-range piece of the wave functions is needed for the matrix element $\langle\psi(k)| U^{\dagger} V_{C} U$ $-V_{C}|\psi(k)\rangle$ in Eq. (11). In Figs. 11 and 12 the zeroenergy wave functions $|\tilde{\psi}(0)\rangle$ and $U|\psi(0)\rangle$ are compared and their short-range features are indeed quantitatively alike. The approximation remains

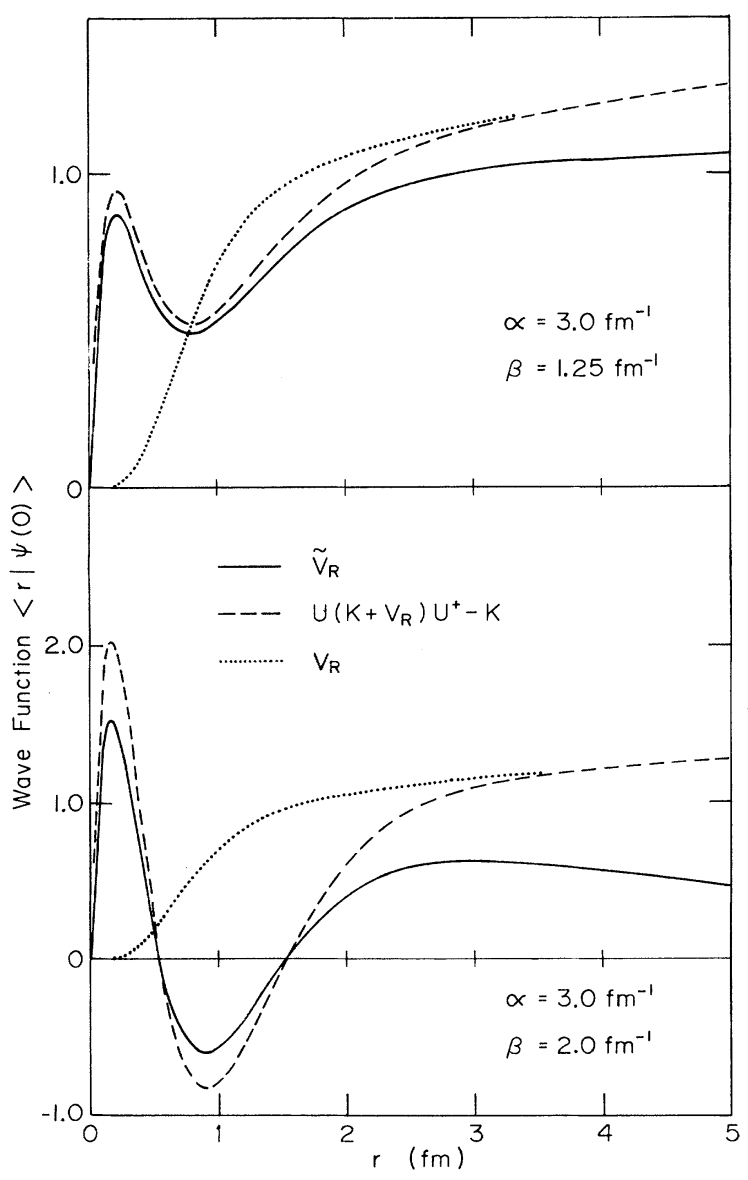

FIG. 11. Zero-energy wave functions in the effectiverange normalization (7). The wave functions $\langle r \mid \tilde{\psi}(0)\rangle$ of $\tilde{V}_{R}$ are obtained according to the method of Appendix B. Results are shown for two potentials $\tilde{V}_{R}$. The point Coulomb potential is used in Eq. (2). The corresponding wave function of $U\left(K+V_{R}\right) U^{\dagger}-K$ are given by $\langle r|U| \psi(0)\rangle$ in terms of the wave function $\langle r \mid \psi(0)\rangle$ of the Reid potential $V_{R}$. good, even if $\tilde{V}_{R}$ supports a bound state, which the potential $U\left(K+V_{R}\right) U^{\dagger}-K$ does not have (case $\alpha=3$ $\mathrm{fm}^{-1}, \beta=2.0 \mathrm{fm}^{-1}$ in Fig. 11). The short-range form of the scattering wave functions is exotic; thus, the radial shape of the nonlocalities in $\tilde{V}_{R}$ are as unusual as those of Ref. 9. It is comforting to note that these strange short-range modifications of the Reid wave function are ruled out by the requirement of charge symmetry. The nonlocalities in $\tilde{V}_{R}$ are of short range $\alpha^{-1}$, but are strong and affect the wave functions up to about 3 fm for $\alpha=3 \mathrm{fm}^{-1}$ and up to about $2 \mathrm{fm}$ for $\alpha=4$ $\mathrm{fm}^{-1}$.

The comparison with the results of exact calculations in Figs. 13 and 14 proves that the approximate relations (11) for $\eta_{p p}(k)$ are very reliable indeed. Relation (11a) accounts for the scattering length and its dependence on $\tilde{V}_{R}$ very well; relation (11b) accounts for the phase shift well

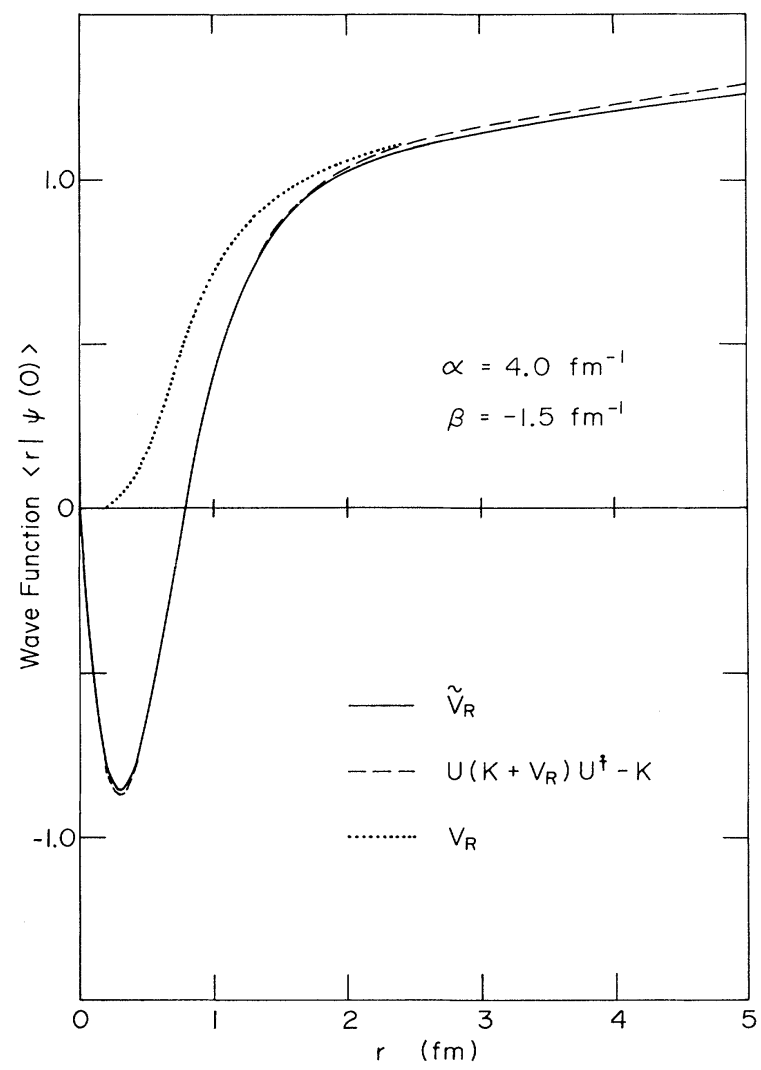

FIG. 12. Zero-energy wave functions in the effectiverange normalization (7). The wave function $\langle r \mid \tilde{\psi}(0)\rangle$ of $\tilde{V}_{R}$ is obtained according to the method of Appendix B. The result is shown for one potential $\tilde{V}_{R}$. The finitesize Coulomb potential is used in Eq. (2). The corresponding wave function of $U\left(K+V_{R}\right) U^{\dagger}-K$ is given by $\langle r|U| \psi(0)\rangle$ in terms of the wave function $\langle r \mid \psi(0)\rangle$ of the Reid potential $V_{R}$. 
at all energies, except if the deviation $\eta_{p p}(k)$ $-\eta_{p p R}(k)$ is large with respect to $\eta_{p p R}(k)$. E.g., this happens at small energies if $\tilde{V}_{R}$ has a bound state as in Fig. 14. In that case the use of Eq. (11a) for the phase shift would be more appropriate. Nevertheless, we stick to Eq. (11b), for its discussion of the phase shift is slightly more transparent. Thus, the relations (11) are so accurate that they can be used as reliable substitutes for exact calculations.

The relations (11) allow us now to understand the trends in the results of Sec. II B by discussing the matrix element $\left\langle\psi(k)\left|U^{\dagger} V_{C} U-V_{C}\right| \psi(k)\right\rangle$. This matrix element depends on the wave function over the range of the unitary transformation $U$. The positive definite and decreasing function $V_{C}$ is integrated (i) with the square of the wave function $U|\psi(k)\rangle$ having an increased amplitude at small distances $r$ as in Figs. 11 and 12 and (ii) with the square of the Reid wave function $|\psi(k)\rangle$ being suppressed at small distances. The two results are

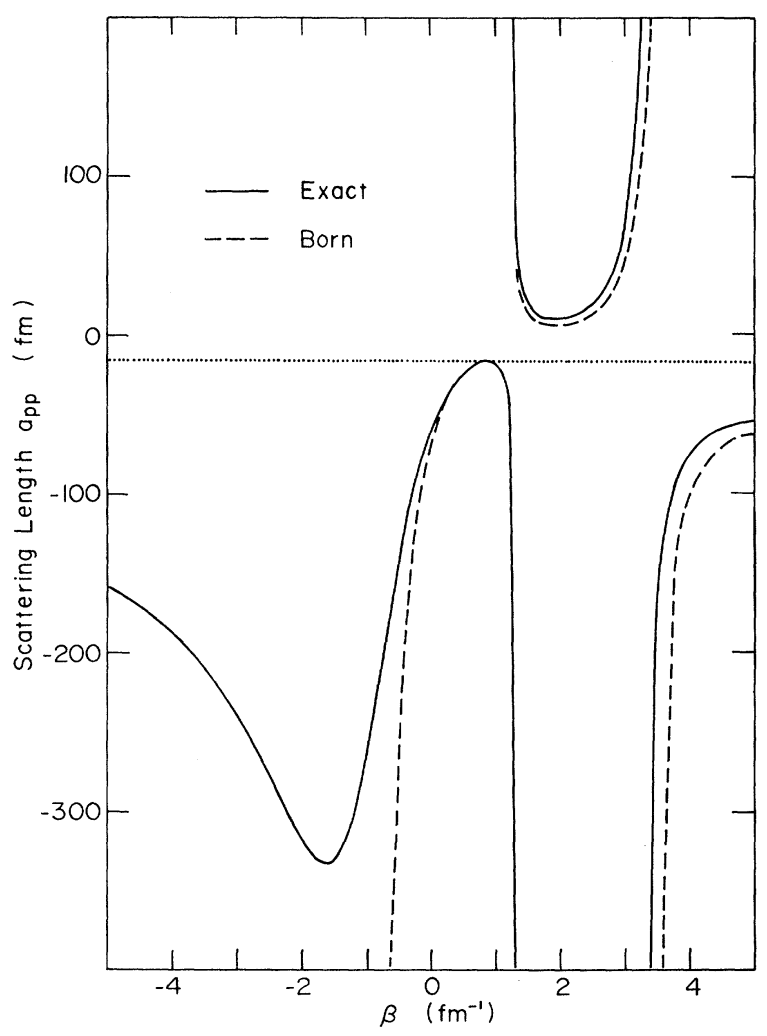

FIG. 13. Scattering length $a_{p p}$ of the Coulomb-subtracted $p p$ phase shifts $\eta_{p p}(k)$ resulting from the potentials $\tilde{V}_{R}$. The point Coulomb potential is taken for $V_{C}$. The unitary transformation (6) is used with the range parameter $\alpha$ being $3 \mathrm{fm}^{-1}$. The results are shown as function of the parameter $\beta$. The scattering length calculated approximatively according to Eq. (11a) is compared with the exact result of Fig. 2 . subtracted for the matrix element (11). The matrix element (11) is positive and therefore always yields purely nuclear scattering results which are more attractive than those of $V_{R}$. This explains the one-sided trend in the Figs. 2, 4, and 7. The matrix element $\left\langle\psi(k)\left|U^{\dagger} V_{C} U-V_{C}\right| \psi(k)\right\rangle$ is especially large for the point Coulomb interaction, since $U|\psi(k)\rangle$, increased at small $r$, effectively exploits the $1 / r$ singularity. In contrast, if $V_{C}$ were constant where $U$ changes the wave function $|\psi(k)\rangle$, the matrix element would be zero. Since the finite-size Coulomb potential $V_{C}^{F}$ is rather smooth over the range of $U$, it is now plausible why the model dependence of the Coulomb-subtracted scattering amplitude obtained with $V_{C}^{F}$ is decreased as compared to the results with the point Coulomb potential $V_{C}^{P}$. The unitary transformation (6) changes its character most rapidly between the $\beta$ values $-1 \mathrm{fm}^{-1}$ and $4 \mathrm{fm}^{-1}$ for the two range parameters $\alpha$ used. We observe that the dramatic variations of the results in Figs. 2 and 3 and 7-9 occur within this parameter range. Outside this range the results still differ from that of the Reid potential but remain rather stable.

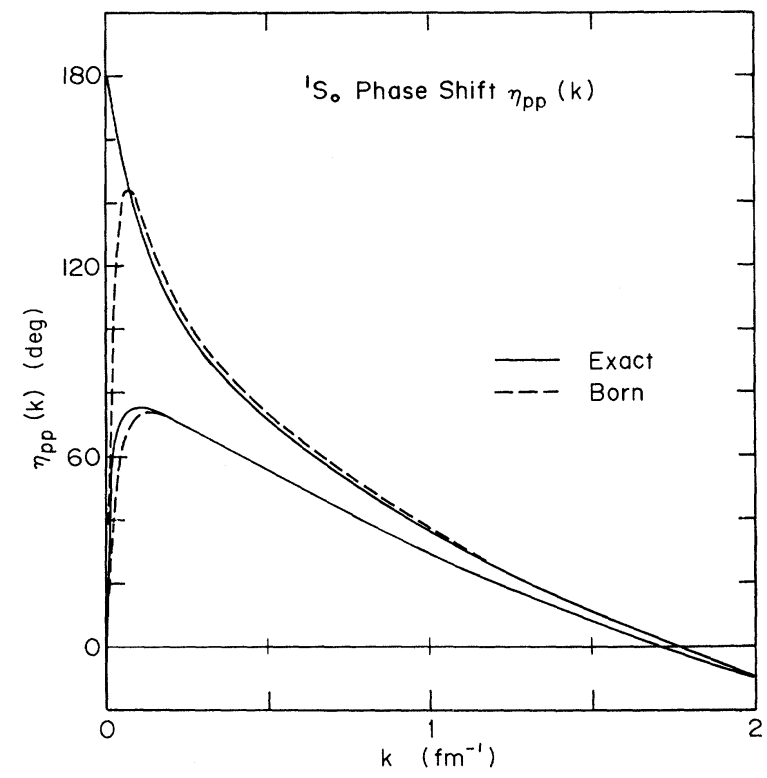

FIG. 14. Coulomb-subtracted $p p$ phase shifts $\eta_{p p}(k)$ resulting from the potentials $\tilde{V}_{R}$. The point Coulomb potential is taken for $V_{C}$. The unitary transformation (6) is used with $\alpha$ being $3 \mathrm{fm}^{-1}$ and $\beta$ being $1.25 \mathrm{fm}^{-1}$ (lower two curves) and $2.00 \mathrm{fm}^{-1}$ (upper two curves). The phase shifts calculated approximately according to Eq. (11b) are compared with the exact results of Fig. 4. Their low-energy approximation could have been improved by using relation (11a) instead of (11b). The phase shifts are plotted as function of the relative momentum $k$. The maximum momentum of $2 \mathrm{fm}^{-1}$ corresponds to $332 \mathrm{MeV}$ energy in the lab system. 
Furthermore, the charge-symmetry constraint can now easily be implemented in the choice of $\tilde{V}_{R}$. If $U$ is chosen to leave the zero-energy wave function of $V_{R}$ unaltered,

$$
U|\psi(0)\rangle=|\psi(0)\rangle,
$$

then, according to Eq. (11a), the Coulomb-subtracted scattering length does not change, i.e., $a_{p p} \simeq a_{p p R}$. Given the range $\alpha^{-1}$ of the unitary transformation (6), the condition (12) is satisfied for one value of $\beta$. For $\alpha=3 \mathrm{fm}^{-1}$ it occurs at $\beta=0.9 \mathrm{fm}^{-1}$, for $\alpha=4 \mathrm{fm}^{-1}$ it occurs at $\beta=1.1$ $\mathrm{fm}^{-1}$. The small parameter range around these special $\beta$ values, whose corresponding potentials $\tilde{V}_{R}$ account for the $n n$ scattering length as well as the Reid potential according to Figs. 2 and 7 , is now understood. Condition (12) can easily be imposed on any unitary transformation. It is a simple practical device to meet the charge-symmetry constraint on the off-shell parametrization of the nucleon-nucleon interaction.

Constraining the potentials $\tilde{V}_{R}$ by condition (12) assures that the fit of $V_{R}$ to the $p p$ data and the $n n$ scattering length will remain close. But let us assume that $n n$ data become as abundant and accurate as $p p$ data and $V_{R}$ accounts for all existing $p p$ and $n n$ data with satisfying accuracy. How can we then preserve such a precious fit in the off-shell changes (2)? The surprising answer is that the single condition (12) is still enough to leave the $n n$ phase shift at all energies approximately unaltered. What is the reason?

With condition (12) satisfied, $U|\psi(k)\rangle-|\psi(k)\rangle$, and therefore also the matrix element (11), remains very small up to about $k=1 \mathrm{fm}^{-1}$. For momenta $k$ larger than $1 \mathrm{fm}^{-1}$ the extra factor $1 / k$ decreases the right side of Eq. (11b) and keeps its value of the order of one degree. Thus, the condition (12) makes $\tilde{V}_{R}$ and $V_{R}$ approximately phase equivalent also with respect to the Coulomb-subtracted phase at all energies. If $V_{R}$ accounts for all $p p$ and $n n$ data, then $\tilde{V}_{R}$ will account for them also, provided $\tilde{V}_{R}$ is chosen in accordance with condition (12). The extra factor $1 / k$ in $(11 \mathrm{~b})$ furthermore ensures that the model dependence of the Coulomb-subtracted scattering amplitude is essentially a lowenergy phenomenon. As shown in Fig. 1, the difference between $\eta_{p p R}^{c}(k)$ and $\eta_{p p R}(k)$ is small at higher energies anyhow.

In this section, we have shown that the Coulomb subtraction in the $p p$ data is quite ambiguous, since it strongly depends on the parametrization of the theoretically unknown short-distance part of the nuclear force. Thus, the isospin symmetries of the nucleon-nucleon interaction cannot be proven $^{24}$ in a model-independent way by comparing $n p$ or $n n$ scattering data with $p p$ scattering data.
However, charge symmetry assumed to hold as a theoretical principle is a very effective off shell constraint in the ${ }^{1} S_{0}$ partial wave. Many potentials $\tilde{V}_{R}$, which fit the $p p$ data as well as the Reid potential but introduce exotic short-range correlations into the low-energy wave functions, are unable to account for the experimentally measured $n n$ scattering length and therefore have to be ruled out. In contrast, the potentials $\tilde{V}_{R}$ of Eq. (2) that are consistent with the simple condition (12) reproduce the $n n$ data as well as $V_{R}$ does at all energies. The off-shell constraint (12) can easily be implemented in the choice of $\tilde{V}_{R}$.

\section{III. ${ }^{1} S_{0}$ NUCLEON-NUCLEON POTENTIALS EQUIVALENT WITH RESPECT TO THE EXPERIMENTAL NEUTRON-NEUTRON DATA}

The standard way of generating off-shell changes in the ${ }^{1} S_{0}$ nucleon-nucleon potential has been to keep the purely nuclear phase shift $\eta_{p p R}(k)$ unaltered. ${ }^{4-7,9-11}$ The potentials $\bar{V}_{R}$,

$$
\bar{V}_{R}=U\left(K+V_{R}\right) U^{\dagger}-K
$$

have this property, and the potentials $\bar{V}_{R}$ and $V_{R}$ reproduce the experimental $n n$ scattering length $a_{n n}$ in exactly the same way. If $U$ is chosen to be of sufficiently short range, $\bar{V}_{R}$ and $V_{R}$ have the theoretically required one pion exchange tail. Thus, $\bar{V}_{R}$ and $V_{R}$ are equally acceptable $n n$ potentials. If we assume charge symmetry to hold, the $\bar{V}_{R}$ and $V_{R}$ can also be taken as hadronic parts for the $p p$ interaction. In fact, they always have been used this way in nuclear-structure calculations. ${ }^{4-7,9-11}$ However, do the potentials $\bar{V}_{R}$ and $V_{R}$ account for the experimental $p p$ data? The Reid potential does; for it was fitted to do so. Does $\bar{V}_{R}$ account for the experimental $p p$ data equally well?

We study the scattering problem for the Hamiltonian $K+V_{C}+\bar{V}_{R}$. The potentials $\bar{V}_{R}$ are most conveniently given in momentum space, and according to the method of Ref. 26 the scattering problem with Coulomb could indeed be solved exactly in momentum space. However, we prefer to rely on the approximate method of Sec. IIC for estimating the phase shifts $\eta_{p p}^{C}(k)$ of $\bar{V}_{R}$. In this way transparent relations between $\eta_{p p}^{c}(k)$ and the phase shift $\eta_{p p R}^{c}(k)$ of the Reid potential $V_{R}$ arise, which can easily be discussed. Using the two-potential formula the potentials $\bar{V}_{R}$ are compared with the potentials $\tilde{V}_{R}$ of Eq. (2). As in Sec. IIC the difference between the purely nuclear potentials $\bar{V}_{R}$ and $\tilde{V}_{R}$ is $U V_{c} U^{\dagger}-V_{c}$. The exact relation

$$
\begin{aligned}
C_{0}{ }^{2}(\gamma) k \cot \eta_{p p}^{C}(k)= & C_{0}{ }^{2}(\gamma) k \cot \eta_{p p R}^{C}(k) \\
& -\frac{M}{\hbar^{2}}\left\langle\boldsymbol{U} \phi(k)\left|\boldsymbol{U} V_{c} U^{\dagger}-V_{C}\right| \bar{\phi}(k)\right\rangle
\end{aligned}
$$


is derived in Appendix A. In Eq. (14) $|\bar{\phi}(k)\rangle$ denotes the scattering states of the Hamiltonian $K+V_{C}+\bar{V}_{R}$ with the asymptotic normalization $(3)$ and $\eta_{p p R}^{c}(k)$ replaced by $\eta_{p p}^{c}(k)$. Equivalent in spirit to Sec. II C, $|\bar{\phi}(k)\rangle$ is approximated by $U|\phi(k)\rangle$ and the phase shifts $\eta_{p p}^{c}(k)$ are obtained entirely in terms of $U$ and of quantities referring to the original Reid potential $V_{R}$ :

$$
\begin{aligned}
C_{0}{ }^{2}(\gamma) k \cot \eta_{p p}^{C}(k) \simeq C_{0}{ }^{2}(\gamma) k \cot \eta_{p p R}^{C}(k) & \\
& +\frac{M}{\hbar^{2}}\left\langle\phi(k)\left|U^{\dagger} V_{c} U-V_{c}\right| \phi(k)\right\rangle, \\
\eta_{p p}^{C}(k)-\eta_{p p R}^{c}(k) \simeq & -\frac{\sin ^{2} \eta_{p p R}^{C}(k)}{k} \frac{1}{C_{0}^{2}(\gamma)} \frac{M}{\hbar^{2}} \\
& \times\left\langle\phi(k)\left|U^{\dagger} V_{C} U-V_{C}\right| \phi(k)\right\rangle .
\end{aligned}
$$

The validity of the crucial approximation $|\bar{\phi}(k)\rangle$

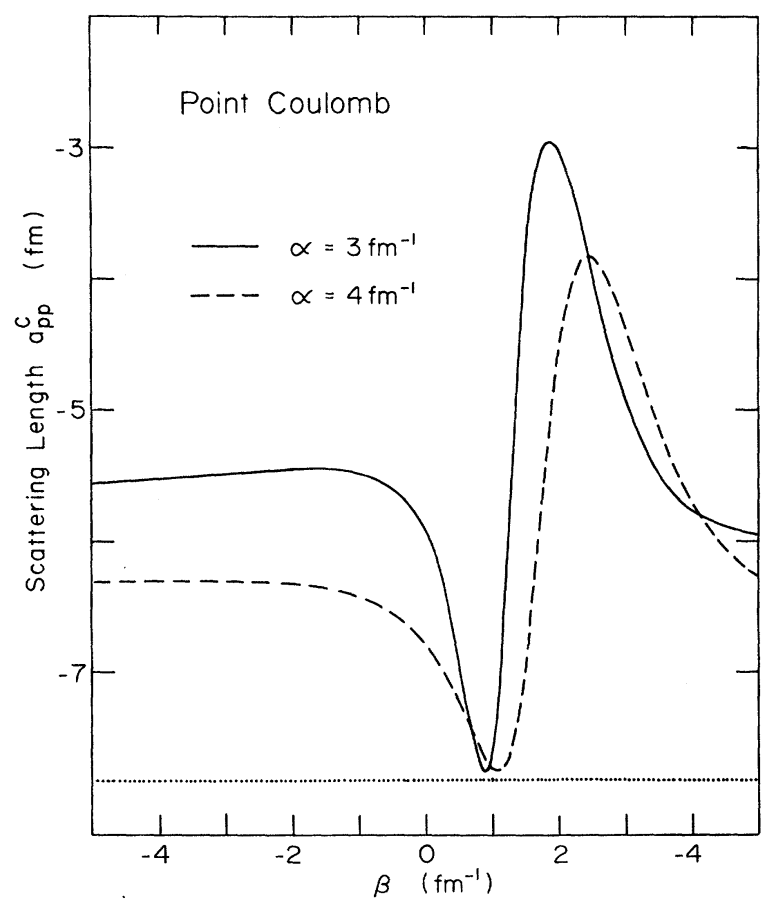

FIG. 15. Scattering length $a_{p p}^{c}$ of the $p p$ phase shifts $\eta_{p p}^{C}(k)$ resulting from the potentials $\bar{V}_{R}$. The approximate relation (15a) is used. The point Coulomb potential is taken for $V_{C}$. The unitary transformation (6) is employed with two different range parameters $\alpha$. The results are shown as function of the parameter $\beta$. The scattering length $a_{p p R}^{C}$ of the untransformed Reid potential is $-7.78 \mathrm{fm}$. It corresponds to the minima of the curves and is slightly less attractive than the experimental value $-7.823 \pm 0.01 \mathrm{fm}$, which is indicated by the dotted line. $\simeq U|\phi(k)\rangle$ has not been checked. There is, however, no apparent reason to believe that the present approximation is less accurate than the equivalent one on which Eqs. (11) are based and which was demonstrated to be very reliable. Thus, we assume (15) also to be trustworthy relations for $\eta_{p p}^{c}(k)$, at least if the changes in the phase shifts are small. For our purposes this is sufficient, since in the case of large changes the potential fit of $V_{R}$ to the experimental data is spoiled anyhow. A drastic deviation of $\eta_{p p}^{C}(k)$ from $\eta_{p p R}^{C}(k)$ and therefore from the experimental ${ }^{1} S_{0} p p$ phase shift is a compelling reason to discard the potential $\bar{V}_{R}$ as unrealistic; the accurate quantitative extent of the disagreement with experiment is not of interest.

Using the point Coulomb and the finite-size Coulomb potentials, respectively, with their corresponding Reid wave functions $|\phi(k)\rangle$ in Eq. (15), the $p p$ phase shifts $\eta_{p p}^{C}(k)$ are calculated for a variety of potentials $\bar{V}_{R}$. Their scattering lengths $a_{p p}^{c}$ obtained from the effective-range expansion (4) of $\eta_{p p}^{c}(k)$ are given in Figs. 15 and 16. The lowenergy phase shifts of some selected potentials $\bar{V}_{R}$ are shown in Fig. 17. In Figs. 18 and 19 the experimental phase shifts at 25 and $50 \mathrm{MeV}$ energy

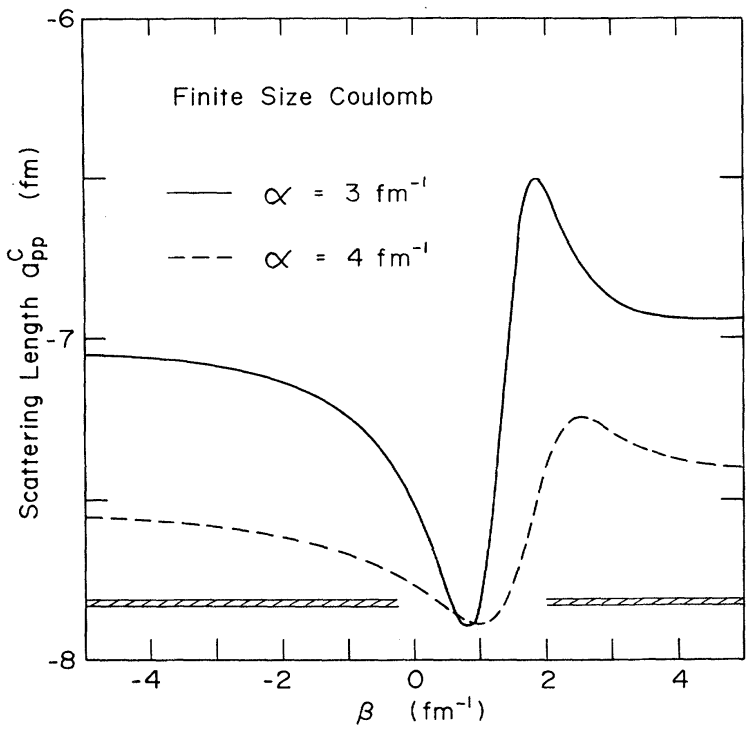

FIG. 16. Scattering length $a_{p p}^{c}$ of the $p p$ phase shifts $\eta_{p p}^{c}(k)$ resulting from the potentials $\bar{V}_{R}$. The approximate relation (15a) is used. The finite-size Coulomb potential is taken for $V_{C}$. The unitary transformation (6) is employed with two different range parameters $\alpha$. The results are shown as function of the parameter $\beta$. The scattering length $a_{p p R}^{C}$ of the untransformed Reid potential is $-7.89 \mathrm{fm}$. It corresponds to the minima of the curves and is slightly more attractive than the experimental value $-7.823 \pm 0.01 \mathrm{fm}$ which is indicated by the cross-hatched strip. 
in the lab system are compared with the corresponding theoretical values. As is evident from the figures, most potentials $\bar{V}_{R}$ are unable to reproduce the experimental $p p$ data and are therefore unacceptable $p P$ potentials. Thus, the experimental $p p$ data and the assumption of charge symmetry are very effective in ruling out numerous off-shell changes of the form (13). The potentials $\bar{V}_{R}$, which correspond to acceptable off-shell changes, are generated from unitary transformations $U$ with $\beta$ values close to $0.9 \mathrm{fm}^{-1}$ for $\alpha=3 \mathrm{fm}^{-1}$ and close to $1.1 \mathrm{fm}^{-1}$ for $\alpha=4 \mathrm{fm}^{-1}$. These transformations

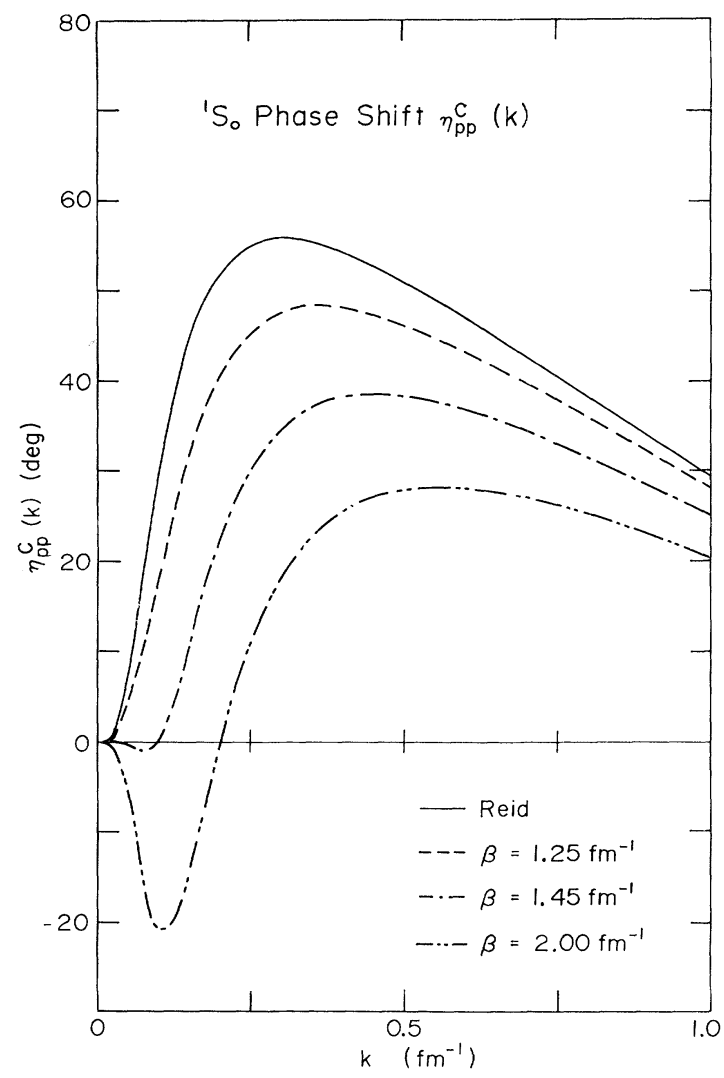

FIG. 17. $p p$ phase shifts $\eta_{p p}^{C}(k)$ resulting from selected potentials $\bar{V}_{R}$. The approximate relation $(15 \mathrm{~b})$ is used. As relation (11b) in Fig. 14, it is not appropriate for the large phase-shift changes at low energies. The phase shifts $\eta_{p p}^{C}(k)$ do not turn negative. The lowenergy approximation could have been improved by using relation (15a) instead of (15b). The point Coulomb potential is taken for $V_{C}$. The unitary transformation (6) is used with $\alpha$ being $3 \mathrm{fm}^{-1}$ and with three different $\beta$ values, $1.25,1.45$, and $2.00 \mathrm{fm}^{-1}$. The corresponding phase shift $\eta_{p p R}^{C}(k)$ of the untransformed Reid potential, which is sufficiently close to the experimental phase shift, is also shown. The phase shifts are plotted as function of the relative momentum $k$. The maximum momentum $1 \mathrm{fm}^{-1}$ corresponds to $83 \mathrm{MeV}$ energy in the lab system. approximately satisfy

$$
U|\phi(0)\rangle=|\phi(0)\rangle \text {. }
$$

They are almost the same as those which satisfy condition (12) and which are consistent with the charge-symmetry constraint for the potentials $\tilde{V}_{R}$. This fact is plausible, since $|\phi(k)\rangle$ and the Coulomb-subtracted wave functions $|\psi(k)\rangle$ are very similar at small relative distances where $U$ acts.

The condition (16) is satisfied at the minima of the theoretical curves of Figs. 15 and 16, and then $a_{p p}^{c} \simeq a_{p p R}^{c}$ holds according to Eq. (15a). But as in Sec. II C, the condition (16) furthermore guarantees that the potentials $\bar{V}_{R}$ preserve the fit of $V_{R}$ to the data; it guarantees that the potentials $\bar{V}_{R}$ and $V_{R}$ remain approximately phase equivalent at all energies. This fact is exemplified by the results of

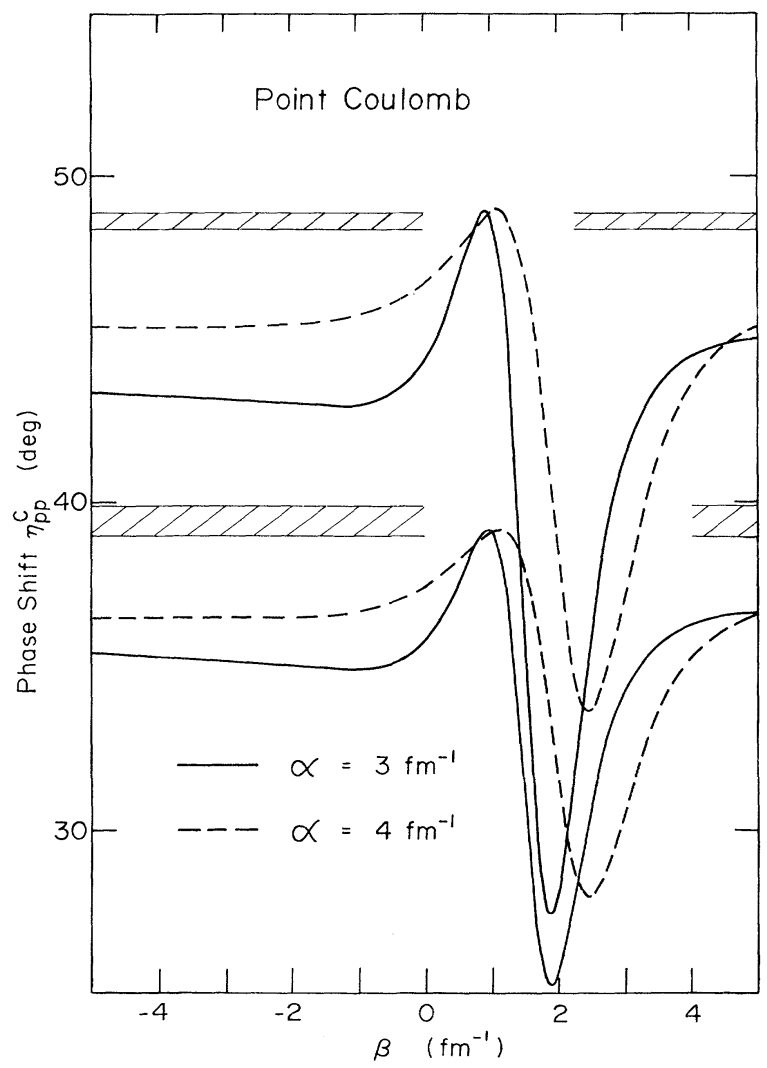

FIG. 18. $p p$ phase shifts $\eta_{p p}^{C}(k)$ at 25 and $50 \mathrm{MeV}$ energy in the lab system resulting from the potentials $\bar{V}_{R}$. The approximate relation $(15 \mathrm{~b})$ is used. The point Coulomb potential is taken for $V_{C}$. The unitary transformation (6) is employed with two different range parameters $\alpha$. The results are shown as function of the parameter $\beta$. The experimental values obtained in Ref. 19 from an energy-independent phase-shift analysis are indicated by the cross-hatched strips. They are $48.60 \pm 0.26^{\circ}$ at $25 \mathrm{MeV}$ and $39.43 \pm 0.44^{\circ}$ at $50 \mathrm{MeV}$. 
Figs. 18 and 19. Thus, the off-shell parametrizations of $\bar{V}_{R}$ are consistent with the experimental $p p$ data and charge symmetry, once condition (16) is met. The condition (16) can easily be incorporated in the unitary transformations $U$ as a constraint. If condition (16) is not fulfilled, the variations of the scattering length $a_{p p}^{c}$ and of the phase shifts $\eta_{p p}^{C}(k)$ go into the direction of repulsion, since the relevant matrix element $\langle\phi(k)| U^{\dagger} V_{C} U$ $\left.-V_{d} \phi(k)\right\rangle$ of Eq. (15) is positive as in Sec. II C. This trend is opposite to that of the potentials $\tilde{V}_{R}$, because there is a crucial sign difference between the relations (11) and (15).

What is the significance of the present results for the nuclear-structure calculations previously performed with the potentials $\bar{V}_{R}$ ? The parameters, which were chosen in Ref. 6 for the potentials $\bar{V}_{R}$, cluster around $\beta=1 \mathrm{fm}^{-1}$. Their corresponding $p p$ phase shifts are therefore mostly in approximate agreement with the experimental values. In the case of the scattering length the results of the potentials $\bar{V}_{R}$ of Ref. 6 are indicated by the crosses in Fig. 20. There are, however, two potentials, $\mathrm{U} 1$ and U2, which do not account for the experimen-

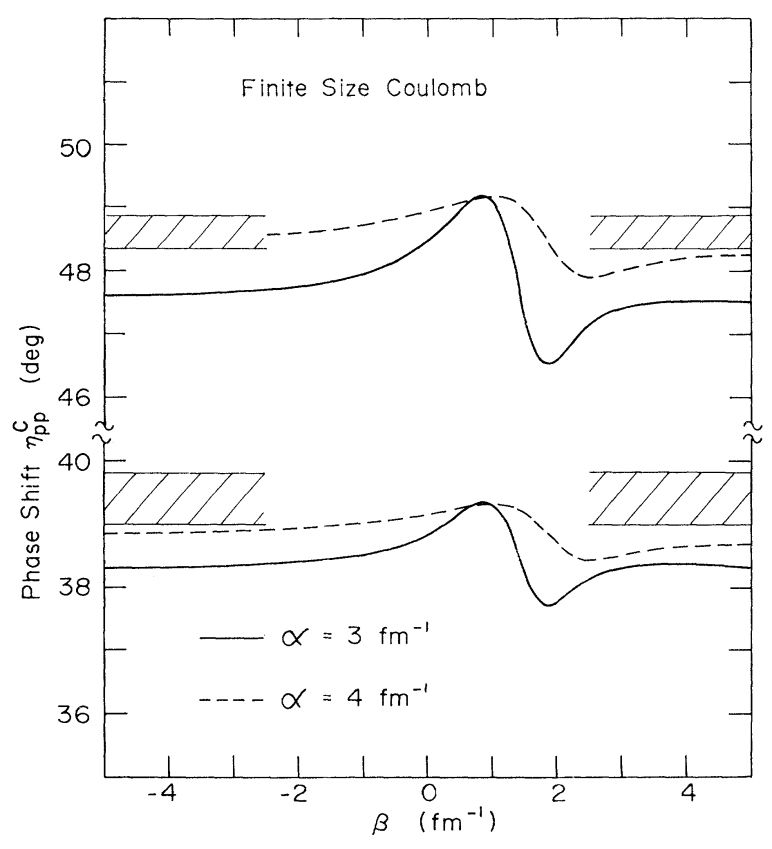

FIG. 19. $p p$ phase shifts $\eta_{p p}^{C}(k)$ at 25 and $50 \mathrm{MeV}$ energy in the lab system resulting from the potentials $\bar{V}_{R}$. The approximate relation $(15 \mathrm{~b})$ is used. The finite-size Coulomb potential is taken for $V_{C}$. The unitary transformation (6) is employed with two different range parameters $\alpha$. The results are shown as function of the parameter $\beta$. The experimental values obtained in Ref. 19 from an energy-independent phase-shift analysis are indicated by the cross-hatched strips. They are $48.60 \pm 0.26^{\circ}$ at $25 \mathrm{MeV}$ and $39.43 \pm 0.44^{\circ}$ at $50 \mathrm{MeV}$. tal scattering length $a_{p p}^{c}$ at all and therefore have to be rejected. It is important to realize that these are the two ${ }^{1} S_{0}$ potentials, which exhibit the most dramatic off-shell effects in nuclear-structure calculations. ${ }^{6,9-11}$ Since these two potentials do not fit the experimental $p p$ data, their offshell effects are of no physical relevancy. The table suggests the remarkable correlation, that those ${ }^{1} S_{0}$ potentials $\bar{V}_{R}$ which account for the experimental $p p$ scattering data allow only moderate variations of nuclear-structure results due to offshell changes. Similar results are expected for the phase-equivalent potentials used in Refs. 5 and 7. The charge symmetry of the nuclear force appears as a very effective off-shell constraint which decreases otherwise disturbingly large off -shell variations to still tolerable magnitudes.

\section{CONCLUSION}

Off-shell changes are generated in the ${ }^{1} S_{0}$ nucleon-nucleon interaction using the Reid soft-core potential and unitary transformations of short range. The Reid potential fits the experimental $p p$ data. ${ }^{17,19}$ It also accounts for the $n n$ scattering length with satisfying accuracy. The off-shell behavior of the Reid potential is varied in two different ways.

First, off-shell changes are performed which preserve the fit to the $p p$ data. However, the Coulomb-subtracted, purely nuclear scattering amplitude is quite model-dependent ${ }^{28}$ and varies strongly with the parametrization of the theoretically unknown short-distance part of the nuclear force. Thus, we have to conclude that the isospin symmetries of the nucleon-nucleon interaction cannot be confirmed in a model-independent way by compar-

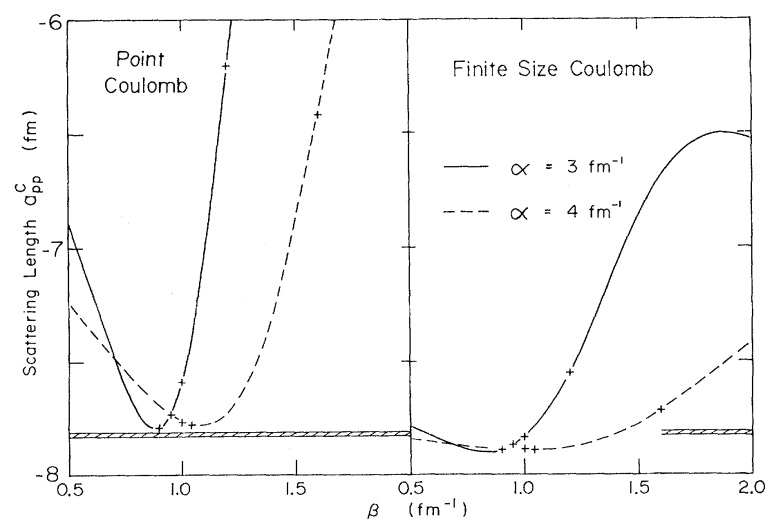

FIG. 20. Scattering length $a_{p p}^{c}$ of the pp phase shifts $\eta_{p p}^{C}(k)$ resulting from the potentials $\bar{V}_{R}$. The results of Figs. 15 and 16 are redrawn and the potentials suggested in Ref. 6 are indicated by crosses. See Figs. 15 and 16 for details. 
TABLE I. Scattering length $a_{p p}^{c}$ of the $p p$ phase shifts $\eta_{p p}^{c}(k)$ and nuclear-structure results obtained with the potentials $\bar{V}_{R}$ of Eq. (13). The special potentials chosen in Ref. 6 are used, their transformation parameters are given in columns 2 and 3 . The scattering length calculated according to Eq. (15a) with the point Coulomb $\left(V_{C}^{P}\right)$ and the finite-size Coulomb $\left(V_{C}^{F}\right)$ potentials is listed in columns 4 and 5 . The variation of the nuclear-matter binding energy per particle, of the ${ }^{16} \mathrm{O}$ binding energy per particle, of the total ${ }^{3} \mathrm{H}$ binding energy, and of the ${ }^{18} \mathrm{O}$ ground-state energy as compared to the Reid potential are given in columns $6-9$, respectively. The results presented in column 6 for U1 to U4 are taken from Fig. 1 of Ref. 6 . Limits on the range of the nuclear-matter energy variation for U5 to U7 can be estimated from Fig. 4 of Ref. 6; the variation should be smaller than $1.5 \mathrm{MeV}$. The results of column 7 are partly taken from Ref. 9, partly calculated for the purposes of this paper. The results of column 8 for the potentials U1, U3, and U4 are given in Ref. 11, Haftel (Ref. 27) calculated the remaining ${ }^{3} \mathrm{H}$ results. The slight different trends for $\mathrm{U} 1$ and $\mathrm{U} 2$ as compared to nuclear matter and ${ }^{16} \mathrm{O}$ are presumably due to still existing inaccuracies in the three-body calculations. The results of column 9 are calculated here using the bare effective shell-model interaction as in Fig. 10. Reference 10 gives the same results for U1 and U6 corrected by core polarization. They are with 0.4 and 1.2 MeV in good agreement with the results of the table. All shifts of the ${ }^{18} \mathrm{O}$ ground-state energy given in the table are much smaller than for the spectrum of Fig. 10. There, the corresponding shift is $11.1 \mathrm{MeV}$. As observed in Ref. 10 and borne out in the table, the off-shell changes in the ${ }^{18} \mathrm{O}$ shell-model spectrum might show different trends compared to other nuclear-structure results. The reason is that the shell-model spectrum is sensitive towards the state dependence in the off-shell variation of the reaction matrix.

\begin{tabular}{|c|c|c|c|c|c|c|c|c|}
\hline $\bar{V}_{R}$ & $\begin{array}{c}\alpha \\
\left(\mathrm{fm}^{-1}\right)\end{array}$ & $\begin{array}{c}\beta \\
\left(\mathrm{fm}^{-1}\right)\end{array}$ & $\begin{array}{l}a_{p p p}^{C}\left(V_{C}^{P}\right) \\
(\mathrm{fm})\end{array}$ & $\begin{array}{l}a_{p p}^{C}\left(V_{C}^{F}\right) \\
\quad(\mathrm{fm})\end{array}$ & $\begin{array}{c}\text { Nuclear matter } \\
\Delta E / A \\
(\mathrm{MeV})\end{array}$ & $\begin{array}{c}{ }^{16} \mathrm{O} \\
\Delta E / A \\
(\mathrm{MeV})\end{array}$ & $\begin{array}{c}{ }^{3} \mathrm{H} \\
\Delta E \\
(\mathrm{MeV})\end{array}$ & $\begin{array}{c}0^{+} \text {ground } \\
\text { state of }{ }^{18} \mathrm{O} \\
\Delta E \\
(\mathrm{MeV})\end{array}$ \\
\hline Reid & & & -7.78 & -7.89 & 0 & 0 & 0 & 0 \\
\hline $\mathrm{U} 1$ & 3.0 & 1.20 & -6.20 & -7.55 & 6.5 & 2.0 & 0.8 & 0.3 \\
\hline U2 & 4.0 & 1.60 & -6.42 & -7.72 & 4.8 & 1.6 & 1.0 & 0.8 \\
\hline U3 & 3.0 & 1.00 & -7.59 & -7.84 & -0.2 & -0.3 & -0.2 & 0.2 \\
\hline $\mathrm{U} 4$ & 4.0 & 1.00 & -7.76 & -7.89 & 1.5 & 0.5 & 0.3 & 0.8 \\
\hline U5 & 4.0 & 1.04 & -7.78 & -7.89 & $(<1.5)$ & 0.4 & 0.2 & 0.6 \\
\hline U6 & 3.0 & 0.90 & -7.79 & -7.89 & $(<1.5)$ & 0.3 & 0.3 & 1.2 \\
\hline U7 & 3.0 & 0.95 & -7.73 & -7.87 & $(<1.5)$ & -0.1 & 0.2 & 0.3 \\
\hline
\end{tabular}

ing $n p$ and $n n$ scattering data with $p p$ scattering data. However, charge symmetry is assumed to hold as a theoretical principle and then the transformed $p p$ potentials are also $n n$ potentials. Most of them are unable to yield the correct value for the $n n$ scattering length and therefore have to be rejected. A simple practical rule is given according to which the off-shell changes consistent with the experimental $n n$ scattering length can be selected. This rule does not allow dramatic variations in the short-range correlations of the low-energy wave functions.

Second, off-shell changes are performed which leave the purely nuclear phase shift and therefore the $n n$ scattering length unaltered. The transformed potentials are acceptable $n n$ potentials. Assuming charge symmetry they are also $p p$ potentials. However, most of them are unable to yield the correct experimental $p p$ phase shift and on this ground have to be rejected. A simple practical rule is given according to which the off-shell changes consistent with the experimental $p p$ data can be selected.

Transformed ${ }^{1} S_{0}$ nucleon-nucleon potentials of the second type have usually been employed in nuclear-structure calculations. Potentials which exhibit the largest off-shell effects are found to be in disagreement with the experimental $p p$ data. Their off-shell effects are therefore of no physical significance. The charge symmetry of the nuclear force is a simple off-shell constraint in the ${ }^{1} S_{0}$ partial wave which, by the conditions (12) and (16), can easily be implemented in unitary transformations and which effectively rules out ${ }^{1} S_{0}$ potentials with large off-shell effects in nuclear structure.

It is a pleasure to thank M. I. Haftel for calculating and communicating the results of column 8 in the table, D. W. L. Sprung for pointing out the deficiency of the Reid soft-core potential as indicated in Ref. 17, B. Giraud for a suggestion which led to the computational technique of Appendix B, and M. Baranger, J. Gillespie, J. W. Negele, J. P. Vary, and H. Walliser for fruitful 
discussions. The author is grateful to C. McKinnis for her help in performing part of the calculations.

\section{APPENDIX A: DERIVATION OF EQS. (10) AND (14)}

The purely nuclear transition matrices,

$$
\begin{aligned}
& T_{i}(\omega)=V_{i}+V_{i}(\omega-K)^{-1} T_{i}(\omega), \\
& \Omega_{i}(\omega)=1+(\omega-K)^{-1} T_{i}(\omega),
\end{aligned}
$$

corresponding to the two potentials $V_{1}=\tilde{V}_{R}$ of (2) and $V_{2}=\bar{V}_{R}$ of Eq. (13) are related by the identity

$$
T_{1}(\omega)=T_{2}(\omega)+\Omega_{2}(\omega *)^{\dagger}\left[V_{1}-V_{2}\right] \Omega_{1}(\omega) .
$$

The two ${ }^{1} S_{0}$ transition matrices in momentum representation $\langle r \mid k\rangle=(2 / \pi)^{1 / 2} \sin k r$ are compared on-shell,

$$
\left\langle k\left|T_{i}\left(\frac{\hbar^{2}}{M} k^{2}+i 0\right)\right| k\right\rangle=-\frac{\hbar^{2}}{M} \frac{2 k}{\pi} \sin \eta_{i}(k) e^{i \eta_{i}(k)} .
$$

The wave functions $\left|\psi_{i}(k)\right\rangle$ in the effective-range normalization (7) are given in terms of the wave operators $\Omega_{i}\left[\left(\hbar^{2} / M\right) k^{2} \pm i 0\right]$,

$$
\left|\psi_{i}(k)\right\rangle=\left(\frac{1}{2} \pi\right)^{1 / 2} \frac{e^{\mp i \eta_{i}(k)}}{\sin \eta_{i}(k)} \Omega_{i}\left(\frac{\hbar^{2}}{M} k^{2} \pm i 0\right)|k\rangle .
$$

Since $\eta_{1}(k)$ and $\left|\psi_{1}(k)\right\rangle$ are denoted by $\eta_{p p}(k)$ and $|\tilde{\psi}(k)\rangle$, respectively, and $\eta_{2}(k)$ and $\left|\psi_{2}(k)\right\rangle$ equal $\eta_{p p R}(k)$ and $U|\psi(k)\rangle$, respectively, and since, fur thermore, the potential difference $V_{1}-V_{2}$ is $U V_{c} U^{\dagger}$ $-V_{c}$, Eq. (10) follows immediately from (A2).

The transition matrices $T_{i}^{c}(\omega)$,

$$
\begin{aligned}
& T_{i}^{C}(\omega)=V_{i}+V_{i}\left(\omega-K-V_{C}^{P}\right)^{-1} T_{i}^{C}(\omega), \\
& \Omega_{i}^{C}(\omega)=1+\left(\omega-K-V_{C}^{P}\right)^{-1} T_{i}^{C}(\omega),
\end{aligned}
$$

include the point-Coulomb part $V_{C}^{P}$ of the Coulomb potential in the propagator. They refer to the two potentials (2) and (13), but the corresponding potentials $V_{i}$ in Eq. (A5) have to consist of the complete interaction except $V_{C}^{P}$, i.e., $V_{1}=\tilde{V}_{R}+V_{C}-V_{C}^{P}$ and $V_{2}=\bar{V}_{R}+V_{C}-V_{C}^{P}$. The transition matrices $T_{i}^{C}(\omega)$ are related by the identity

$$
T_{1}^{C}(\omega)=T_{2}^{C}(\omega)+\Omega_{2}^{C}(\omega *)^{\dagger}\left[V_{1}-V_{2}\right] \Omega_{1}^{C}(\omega) .
$$

The two ${ }^{1} S_{0}$ transition matrices in the representation of the regular Coulomb wave functions $\left\langle r \mid f^{C}(k)\right\rangle$
$=(2 / \pi)^{1 / 2} F_{0}(\gamma, k r)$ are compared on shell

$$
\begin{aligned}
\left\langle f^{c}(k)\left|T_{i}^{c}\left(\frac{\hbar^{2}}{M} k^{2}+i 0\right)\right| f^{c}(k)\right\rangle & \\
& =-\frac{\hbar^{2}}{M} \frac{2 k}{\pi} \sin \eta_{i}^{C}(k) e^{i \eta_{i}^{C}(k)} .
\end{aligned}
$$

The wave functions $\left|\phi_{i}(k)\right\rangle$ in the effective-range normalization (3) are given in terms of the wave operators $\Omega_{i}^{C}\left[\left(\hbar^{2} / M\right) k^{2} \pm i 0\right]$,

$$
\begin{aligned}
& \left|\phi_{i}(k)\right\rangle \\
& \quad=\left(\frac{1}{2} \pi\right)^{1 / 2} \frac{C_{0}(\gamma) e^{\mp i \eta C_{i}(k)}}{\sin \eta_{i}^{C}(k)} \Omega_{i}^{C}\left(\frac{\hbar^{2}}{M} k^{2} \pm i 0\right)\left|f^{C}(k)\right\rangle
\end{aligned}
$$

Since $\eta_{1}^{C}(k)$ and $\left|\phi_{1}(k)\right\rangle$ equal $\eta_{p \phi R}^{c}(k)$ and $U|\phi(k)\rangle$, respectively, and $\eta_{2}^{C}(k)$ and $\left|\phi_{2}(k)\right\rangle$ are denoted by $\eta_{p p}^{c}(k)$ and $|\bar{\phi}(k)\rangle$, respectively, since, furthermore, the potential difference $V_{1}-V_{2}$ is $U V_{c} U^{\dagger}$ $-V_{c}$, Eq. (14) follows immediately from (A6).

\section{APPENDIX B: CALCULATION OF THE WAVE FUNCTIONS $|\tilde{\psi}(k)\rangle$ OF $\tilde{V}_{R}$ IN CONFIGURATION SPACE}

The method of Ref. 22 solves the LippmanSchwinger equation (A1a) in momentum space. It is very suitable for the potential $\tilde{V}_{R}$ which has a complicated momentum dependence. It yields the corresponding half-shell transition matrix $\left\langle k^{\prime}\left|T_{1}\left[\left(\hbar^{2} / M\right) k^{2}+i 0\right]\right| k\right\rangle$ at mesh points $k^{\prime}$. From the half-shell transition matrix the wave function $\langle r \mid \tilde{\psi}(k)\rangle$ has to be obtained by integration.

$$
\begin{aligned}
\langle r \mid \tilde{\psi}(k)\rangle= & \frac{e^{-i \eta_{p p}(k)}}{\sin \eta_{p p}(k)} \\
\times & \times \sin k r+\int_{0}^{\infty} d k^{\prime} \frac{\sin k^{\prime} r}{\left(\hbar^{2} / M\right)\left(k^{2}-k^{\prime 2}+i 0\right)} \\
& \left.\times\left\langle k^{\prime}\left|T_{1}\left(\frac{\hbar^{2}}{M} k^{2}+i 0\right)\right| k\right\rangle\right] .
\end{aligned}
$$

Using (B1) it is difficult to achieve accurate results for $\langle r \mid \tilde{\psi}(k)\rangle$ over a large range of $r$, even if the half-shell transition matrix is known at a great number of mesh points. However, the accuracy is dramatically improved by comparing $\langle r \mid \tilde{\psi}(k)\rangle$ with an auxiliary wave function $\left\langle r \mid \psi_{\text {aux }}(k)\right\rangle$, which is analytically known, has the same phase shift as $\langle r \mid \tilde{\psi}(k)\rangle$ at the energy of interest and whose transition matrix $T_{\text {aux }}(\omega)$ can easily be given. The difference between the wave functions $\langle r \mid \tilde{\psi}(k)\rangle$ and $\left\langle r \mid \psi_{\text {aux }}(k)\right\rangle$ is then obtained by the singularity-

free relation

$$
\langle r \mid \tilde{\psi}(k)\rangle=\left\langle r \mid \psi_{\text {aux }}(k)\right\rangle-\frac{2 k}{\pi} \int_{0}^{\infty} d k^{\prime} \frac{\sin k^{\prime} r}{k^{2}-k^{\prime 2}} \frac{\left\langle k^{\prime}\left|T_{1}\left[\left(\hbar^{2} / M\right) k^{2}+i 0\right]-T_{\mathrm{aux}}\left[\left(\hbar^{2} / M\right) k^{2}+i 0\right]\right| k\right\rangle}{\left\langle k\left|T_{1}\left[\left(\hbar^{2} / M\right) k^{2}+i 0\right]\right| k\right\rangle} .
$$


For the calculations of Sec. IIC the wave functions and transition matrix of the Yamaguchi potential with the range parameter $\mu$ chosen to be $0.7 \mathrm{fm}^{-1}$ are used as auxiliary quantities. Then Eq. (B2) takes on the form

$$
\langle r \mid \tilde{\psi}(k)\rangle=\cot \eta_{p p}(k) \sin k r+\cos k r-e^{-\mu r}-\frac{2 k}{\pi} \int_{0}^{\infty} d k^{\prime} \frac{\sin k^{\prime} r}{k^{2}-k^{\prime 2}}\left[\frac{\left\langle k^{\prime}\left|T_{1}\left[\left(\hbar^{2} / M\right) k^{2}+i 0\right]\right| k\right\rangle}{\left\langle k\left|T_{1}\left[\left(\hbar^{2} / M\right) k^{2}+i 0\right]\right| k\right\rangle}-\frac{\mu^{2}+k^{2}}{\mu^{2}+k^{\prime 2}}\right] .
$$

${ }^{*}$ Work supported in part through funds provided by the U.S. Atomic Energy Commission under Contract No. AT(11-1)-3069.

$\dagger$ Permanent address: Department of Theoretical Physics, Technical University, Appelstrasse 1, 3000 Hannover, Germany.

${ }^{1}$ M. Baranger, B. Giraud, S. K. Mukhopadhyay, and P. U. Sauer, Nucl. Phys. A138, 1 (1969).

${ }^{2}$ M. I. Haftel, Phys. Rev. Lett. 25, 120 (1970).

${ }^{3}$ P. U. Sauer, Nucl. Phys. A170, 497 (1971).

${ }^{4} \mathrm{~F}$. Tabakin, in The Two-Body Force in Nuclei, edited by S. M. Austin and G. M. Crawley (Plenum, New York, 1972), p. 101, and references therein.

${ }^{5}$ F. Coester, S. Cohen, B. Day, and C. M. Vincent, Phys. Rev. C 1 , 769 (1970).

${ }^{6}$ M. I. Haftel and F. Tabakin, Phys. Rev. C $\underline{3}, 921$ (1971).

${ }^{7}$ C. W. Wong and T. Sawada, Ann. Phys. (N.Y.) $\underline{72}, 107$ (1972).

${ }^{8}$ P. U. Sauer, Ann. Phys. (N.Y.) 80, 242 (1973).

${ }^{9}$ M. I. Haftel, E. Lambert, and P. U. Sauer, Nucl. Phys. A192, 225 (1972).

${ }^{10}$ H. C. Pradhan, P. U. Sauer, and J. P. Vary, Phys. Rev. C 6 , 407 (1972).

${ }^{11}$ E. P. Harper, Y. E. Kim, and A. Tubis, Phys. Rev. C $\underline{6}, 1601$ (1972).

${ }^{12}$ P. U. Sauer and J. A. Tjon, Nucl. Phys. A216, 541 (1973).

${ }^{13}$ H. P. Noyes, Annu. Rev. Nucl. Sci. 22 , 465 (1972), and references therein.

${ }^{14}$ E. M. Henley and D. H. Wilkinson, in Few Particle Problems in the Nuclear Interaction, edited by I. Šlaus, S. A. Moszkowski, P. P. Haddock, and W. T. H. van Oers (North-Holland, Amsterdam, 1972), p. 242 , and references therein.

${ }^{15}$ E. M. Henley, in Isospin in Nuclear Physics, edited by D. H. Wilkinson (North-Holland, Amsterdam, 1969), p. 17.

${ }^{16} \mathrm{P}$. U. Sauer and H. Walliser, in Contributions to the International Conference on Few Body Problems in Nuclear and Particle Physics, Quebec, 1974 (to be published)

${ }^{17}$ R. V. Reid, Ann. Phys. (N.Y.) 50, 411 (1968). Vacuum polarization has an appreciable effect on the lowenergy $p p$ phase shifts. Since the Reid soft-core potential was fitted without vacuum polarization, it fails to reproduce the experimental phase shifts when vacuum polarization is added [M. W. Kermode and D. W. L. Sprung, Nucl. Phys. A135, 535 (1969)]. As stated in Sec. I, vacuum polarization is deliberately also neglected here. The conclusions of this paper are, however, not affected by this omission, and surely, the present study could be repeated with vacuum polarization included as additional electromagnetic interaction. The author is grateful to D. W. L. Sprung for drawing his attention to the problem of vacuum polarization in the context of the Reid soft-core potential.

${ }^{18} \mathrm{M}$. Abramowitz, in Handbook of Mathematical Functions, edited by M. Abramowitz and I. A. Stegun (Dover, New York, 1965), p. 538.

${ }^{19}$ M. H. MacGregor, R. A. Arndt, and R. M. Wright, Phys. Rev. 182, 1714 (1969).

${ }^{20}$ H. P. Noyes and H. M. Lipinski, Phys. Rev. C $\underline{4}, 995$ (1971).

${ }^{21}$ M. D. Miller, M. S. Sher, P. Signell, N. R. Yoder, and D. Marker, Phys. Lett. 30B, 157 (1969).

${ }^{22}$ G. E. Brown, A. D. Jackson, and T. T. S. Kuo, Nucl. Phys. A133, 481 (1969); M. I. Haftel and F. Tabakin, ibid. A158, 1 (1970).

${ }^{23}$ N. Auerbach, J. Hüfner, A. K. Kerman, and C. M. Shakin, Rev. Mod. Phys. 44, 48 (1972).

${ }^{24}$ P. U. Sauer, Phys. Rev. Lett. 32, 626 (1974).

${ }^{25}$ P. U. Sauer, Nucl. Phys. A150, 467 (1970).

${ }^{26}$ C. M. Vincent and S. C. Phatak, Phys. Rev. C $\underline{10}, 391$ (1974).

${ }^{27}$ M. I. Haftel, private communication.

${ }^{28}$ After the completion of this work the English translation of H. Kumpf [Sov. J. Nucl. Phys. 17, 602 (1973)] appeared. Kumpf uses methods and he addresses himself with problems similar to those of the present paper. 